\title{
Genetic polymorphisms of MBL2 and tuberculosis susceptibility: a meta-analysis of 22 case-control studies
}

\author{
Yan $\mathrm{CaO}^{1}$, Xinjing Wang ${ }^{1}$, Zhihong $\mathrm{CaO}^{1}$, Chunyan $\mathrm{Wu}^{2}$, Dongmei $\mathrm{Wu}^{2}$, Xiaoxing Cheng ${ }^{1}$
}

\author{
${ }^{1}$ Key Laboratory of Tuberculosis Prevention and Treatment, and Beijing Key Laboratory \\ of New Techniques of Tuberculosis Diagnosis and Treatment, Institute \\ of Tuberculosis, the $309^{\text {th }}$ Hospital, Beijing, China \\ ${ }^{2}$ Tangshan Tuberculosis Hospital, Tangshan, Hebei, China \\ Submitted: 25 June 2016 \\ Accepted: 21 September 2016
}

Arch Med Sci 2018; 14, 6: 1212-1232

DOI: https://doi.org/10.5114/aoms.2017.65319

Copyright $\odot 2017$ Termedia \& Banach

\author{
Corresponding author: \\ Xiaoxing Cheng MB, PhD \\ Division of Research \\ Institute of Tuberculosis \\ 309 Hospital \\ 17 Hei Shan Hu Road \\ Haidian \\ Beijing 100091, China \\ Phone/fax: +86 1051520496 \\ E-mail:xcheng79@outlook. \\ com
}

\begin{abstract}
Introduction: The association of mannose-binding lectin gene (MBL2) polymorphisms with tuberculosis susceptibility was inconclusive. In this study, a meta-analysis of 22 case-control studies was carried out to assess the effect of MBL2 polymorphisms on tuberculosis risk.

Material and methods: A search was performed in Embase, PubMed and Web of Science up to Sep 30, 2015. Odds ratio (OR) and 95\% confidence interval $(95 \% \mathrm{Cl})$ were used to assess the association. Statistical analyses were performed using STATA 12.0 software.

Results: rs 1800451 was associated with a decreased tuberculosis risk in the allele model (C vs. A: OR $=0.93,95 \% \mathrm{Cl}: 0.86-1.00, p=0.050)$. In analyses stratified by ethnicity, rs7096206 (C/G: OR $=1.31,95 \% \mathrm{Cl}: 1.10-1.57$, $p=0.003 ; \mathrm{GG}$ vs. $\mathrm{GC}+\mathrm{CC}: \mathrm{OR}=0.69,95 \% \mathrm{Cl}: 0.56-0.85, p<0.001)$ and $\mathrm{A} / \mathrm{O}(\mathrm{O} / \mathrm{A}: \mathrm{OR}=1.34,95 \% \mathrm{Cl}: 1.10-1.64, p=0.004)$ were associated with tuberculosis risk in Asians, $A / O$ (AA vs. $A O+O O: O R=0.71,95 \% \mathrm{Cl}: 0.51-0.99$, $p=0.041$ ) and rs1800451 (AC vs. AA + CC: OR $=2.70,95 \% \mathrm{Cl}: 1.27-5.74$, $p=0.010$ ) were associated with tuberculosis risk in Americans, and rs 1800451 (C/A: $\mathrm{OR}=0.92,95 \% \mathrm{Cl}: 0.86-0.99, p=0.035$ ) was associated with tuberculosis risk in Africans. Additionally, rs 1800450 (B/A: OR $=0.42,95 \% \mathrm{Cl}: 0.25-$ $0.72, p=0.001)$ was associated with tuberculosis risk in Europeans.

Conclusions: The MBL2 rs 1800451 polymorphism is associated with decreased TB risk in the general population, and A/O, rs7096206, rs 1800450 and rs1800451 are likely to be associated with the risk for some specific ethnic groups.
\end{abstract}

Key words: tuberculosis, gene polymorphism, susceptibility.

\section{Introduction}

Mycobacterium tuberculosis (TB) is one of the most common infectious diseases and ranks as the leading cause of mortality worldwide, with approximately 9 million new cases and 1.5 million deaths globally in 2013. According to the latest World Health Organization (WHO) report, the greatest burden of disease falls in developing countries, with approximately $56 \%$ of new cases occurring in the South-East Asia and Western Pacific regions [1]. It is well known that the outcome of infection with $M$. tuberculosis may be influenced by many factors, such as smoking history, physical condition, environmental and host genetic factors [2]. Recently, there have been various studies reporting that host genetic factors may 
play an important role in TB susceptibility, which includes single nucleotide polymorphisms (SNPs) as a major factor. Multiple candidate genes have been investigated to determine the relationship between SNPs and TB risk, including TIRAP [3], VDR [4], P2X7 [5], and $M B L 2$ [6].

The $M B L 2$ gene codes for the complement factor mannose binding lectin $(\mathrm{MBL})$, which can bind through multiple lectin domains to the carbohydrate moieties present in a wide variety of bacteria, viruses and fungi. Upon binding, MBL-associated serine proteases (MASPs) are activated to initiate the lectin pathway of the complement system, opsonizing and facilitating phagocytosis of micro-organisms by macrophages [7]. MBL2 deficiency may be advantageous in resistance against mycobacteria by reducing opsonization. MBL2 also plays a role in the regulation of inflammatory cytokines released by monocytes and enhances tolllike receptor (TLR) 2 and TLR6 signaling in response to microbial infection, and hence may affect the inflammation severity or disease progression [8].

Previous studies have suggested that certain SNPs within the promoter region and structural region of the $M B L 2$ gene affect the formation of $\mathrm{MBL}$ multimer and serum MBL concentration [9]. The reduction of $M B L$ multimer results in impaired binding with the ligand and the increased likelihood of being degraded by metalloproteinase. Three SNPs (rs1800450, rs5030737 and rs1800451) in exon 1 of the MBL2 gene give rise to amino acid substitutions, which disrupt the collagenous structure and the formation of functional oligomers. These three SNPs, collectively designated as "AO" polymorphisms, while the wild-type allele is described as allele " $A$ " and the mutant allele as " $O$ ", indicate the presence of one or more mutant alleles in either rs1800450, rs5030737 or rs1800451. The heterogeneous-type A/O correlates with low MBL levels in the serum and the homologous-type $\mathrm{O} / \mathrm{O}$ with almost undetectable MBL levels. The other SNPs, rs11003125, rs7096206, and rs7095891, also have been found in the promoter and $5^{\prime}$ untranslated region, and the $X$ variant shows negatively regulated transcriptional activity and results in reduced serum MBL levels [10]. Several studies have evaluated the relationship of these polymorphisms with TB risk in black people, Asians, and Caucasians. However, the results obtained are controversial. Considering the critical role of the $M B L 2$ gene in the pathogenesis of tuberculosis and the fact that a small sample size may lack the power to provide comprehensive conclusions, we performed a meta-analysis to investigate the association between $M B L 2$ gene polymorphisms and TB susceptibility. To our knowledge, this is the most comprehensive meta-analysis to investigate the associations of the $M B L 2$ gene polymorphisms and TB risk.

\section{Material and methods}

\section{Literature search}

A systematic search was conducted using the databases of the US National Institutes of Health (PubMed), Web of Science and Embase databases, with the following combination of search terms: 'Mycobacterium tuberculosis' OR 'tuberculosis' AND 'polymorphism' OR 'variant' OR 'genotype' OR 'allele' OR 'mutation' OR 'single nucleotide polymorphism' AND 'MBL' OR 'mannose-binding lectin' OR 'mannose-binding protein'. To identify additional eligible articles, the relevant published articles and review articles were also identified by hand searching. The search in these databases was limited to articles relating to humans, covering all relevant English and Chinese language publications published up to March 2016.

The studies identified in our meta-analysis met all of the following criteria: (1) studies had to assess the association between MBL2 polymorphisms rs5030737, rs1800450, rs1800451, A/O, rs11003125, rs7096206, rs7095891 and tuberculosis risk; (2) unrelated case-control studies or cohort design, and studies included available genotype frequencies to calculate odds ratio (OR) and $95 \%$ confidence interval $(\mathrm{Cl})$; (3) independent studies using original data. Studies were excluded for the following criteria: (1) studies not providing genotype distribution or allele frequency data; (2) reviews or case reports, case studies without control subjects; (3) duplicated previous publications.

\section{Data extraction}

Two investigators (CY and WXJ) independently performed the required data extraction, and then conducted group discussion to resolve the disagreements. The following data were extracted from each study: publication year, name of first author, country, ethnicity, genotyping method, method of diagnosis of cases, tuberculosis type, number of cases and controls, numbers and mean age of cases and controls, genotype and allele frequencies for cases and controls, and HIV status of cases and controls.

\section{Statistical analysis}

Hardy-Weinberg equilibrium (HWE) was assessed among the control population for each study using the Hardy-Weinberg Equilibrium Online Calculator (http://www.changbioscience.com/ genetics/hardy.html). A $p$-value of $>0.05$ was considered to meet HWE.

All statistical analyses were performed using Stata statistical software 12.0 (Stata Corporation, College Station, TX, USA), with two-sided $p$-values. The OR and its corresponding $95 \% \mathrm{Cl}$ were calculated to assess the strength of association 
between MBL2 polymorphism and the TB risk. The pair-wise differences were analyzed to indicate the best genetic models as suggested by Thakkinstian et al. [11]. Data were then pooled using the best model. Ethnicity was adopted to carry out the stratified analysis, when data were available.

The significance of pooled ORs was measured by the Z-test $(p<0.05$ was considered statistically significant). The heterogeneity assumption was tested by the $\chi^{2}$-based Q-statistic and Higgins' $P$ test. If $p<$ 0.10 , the heterogeneity was considered statistically significant, and then the RE model was used. The heterogeneity was considered significant if $R>50 \%$, then ORs were pooled according to the random effect model (Mantel-Haenszel method) [12]. Otherwise the fixed effect model was used (DerSimonian-Laird method) [13]. Meta-regression was performed to detect the source of heterogeneity. Publication bias was evaluated by examining the Begg's funnel plots and Egger's linear regression test $[14,15]$.

\section{Results}

\section{Characteristics of studies}

Our initial search identified 250 articles according to the search terms (PubMed: 56; Embase: 89; Web of Science: 105). One hundred and thirty-two

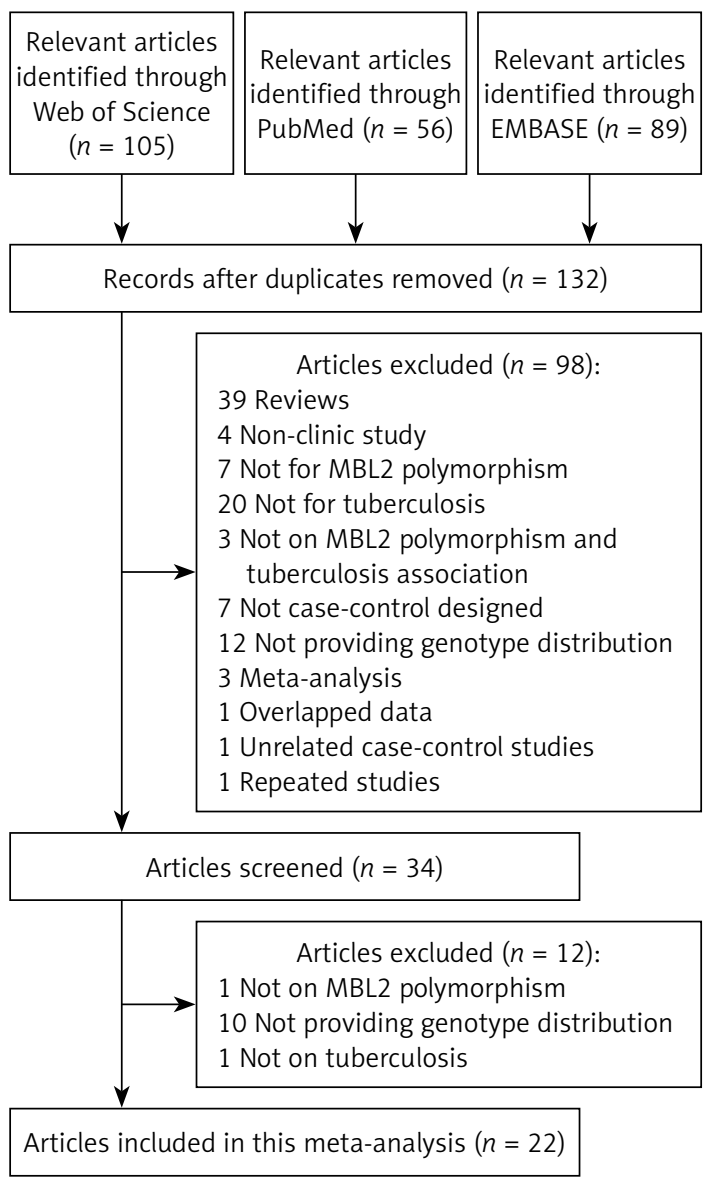

Figure 1. Flowchart for study selection abstracts were retrieved for more detailed evaluation after removing duplicates. Thirty-four articles addressing the association of MBL2 polymorphisms and TB were identified. After reviewing the full text, 12 articles were excluded (1 was excluded due to not being on $M B L 2$ polymorphism; 1 was excluded due to not being on tuberculosis; 10 were excluded due to not providing genotype distribution). Finally, a total of 22 case-control studies that consisted of a total of 7056 tuberculosis patients and 7764 control subjects were included in this meta-analysis [16-37]. Figure 1 provides the detailed screening process. Among them, 9 were performed in an Asian population, 2 were performed in an American population, 6 were performed in a European population and 5 were performed in an African population. The study of Soborg et al. 2003 has mixed data with 59 Europeans, 26 Asians, 17 Africans and 7 Inuit, so it is not included in the subgroup analysis. The characteristics of each included study are listed in Table I.

\section{Quantitative data synthesis}

\section{MBL2 rs7096206 polymorphism}

Six case-control studies (3154 cases and 3441 controls) on the relationship between the rs7096206 polymorphism and the risk of TB were included in the meta-analysis. For rs7096206, the estimated OR1 (CC vs. GG), OR2 (GC vs. GG) and OR3 (CC vs. GC) were 0.964 (95\% Cl: 0.6891.347), 1.290 (95\% Cl: $1.004-1.657)$ and 0.828 (95\% Cl: 0.587-1.168) (Table II). Thus, we mainly pooled the OR for allele comparison and the recessive genetic model in the subgroup analysis by ethnicity (Table III). The pooled examination showed no significant association between rs7096206 polymorphism and the risk of tuberculosis (C/G: OR $=1.15,95 \% \mathrm{Cl}: 0.97-1.36, p=$ $0.100, p^{a}=0.061 ; \mathrm{GG}$ vs. GC + CC: OR $=0.80$, $95 \%$ Cl: 0.64-1.01, $p=0.058, p^{a}=0.010$ ) (Table III). The results of subgroup analysis based on ethnicity indicated that the rs7096206 C allele increased susceptibility to tuberculosis risk in Asian populations, but not in American and African populations (C/G: OR $=1.31,95 \% \mathrm{Cl}: 1.10-1.57, p=0.003$, $p^{a}=0.634 ; \mathrm{GG}$ vs. GC + CC: OR $=0.69,95 \% \mathrm{Cl}$ : $0.56-0.85, p<0.001, p^{a}=0.381$ ) (Table III, Figure 2). For the subgroup analysis by the genotyping methods, the recessive genetic model (GG vs. GC + CC: $\mathrm{OR}=0.76,95 \% \mathrm{Cl}: 0.59-0.96, p=0.022$, $p^{a}=0.152$ ) remained statistically significant in polymerase chain reaction sequence-specific primer (PCR-SSP) studies (Table III).

\section{MBL2 rs11003125 polymorphism}

Four case-control studies (2370 cases and 2760 controls) on the relationship between the 


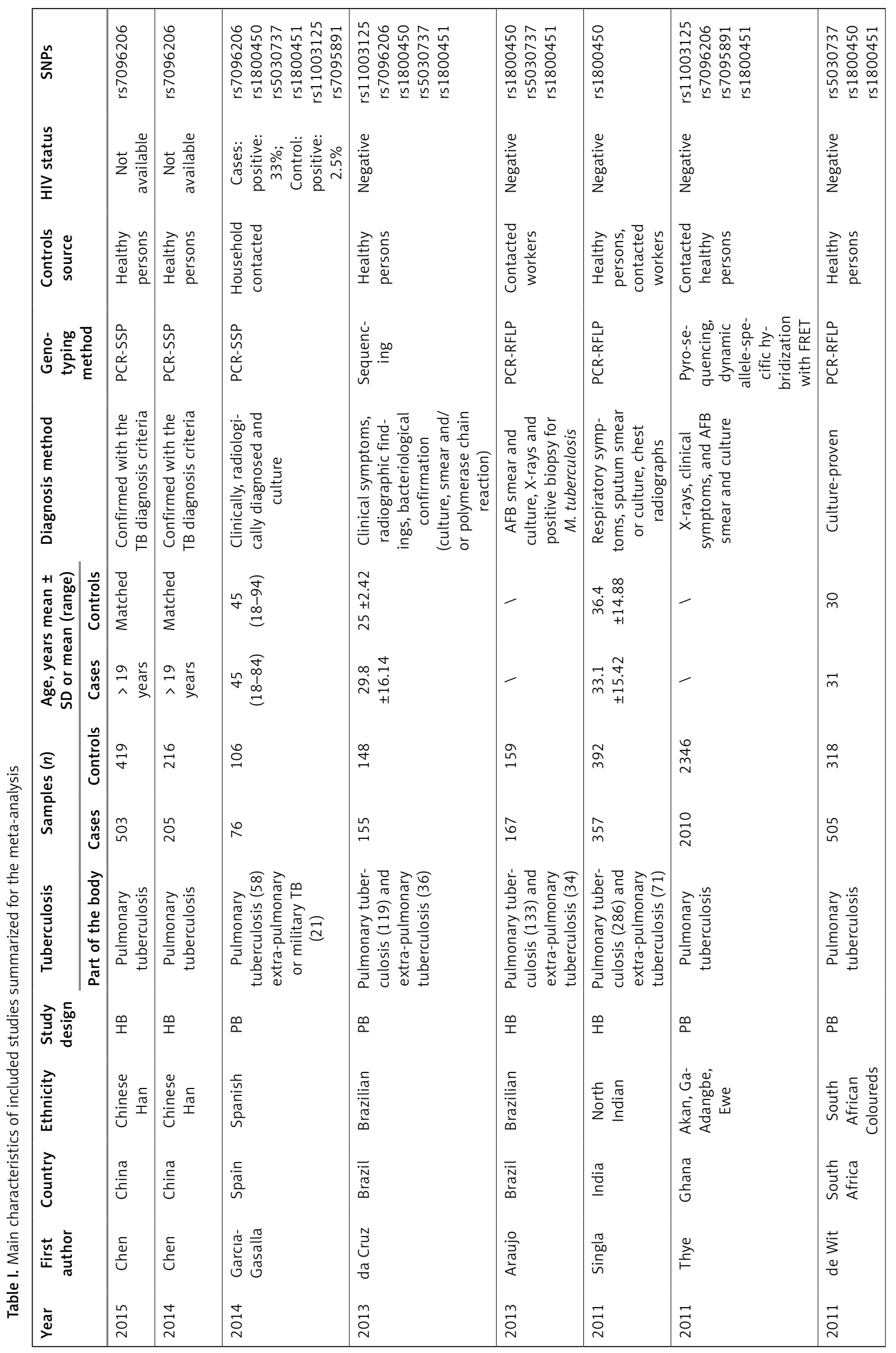




\begin{tabular}{|c|c|c|c|c|c|c|c|}
\hline$\sum_{n}^{n}$ & 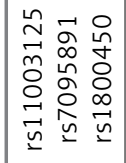 & 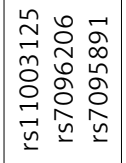 & 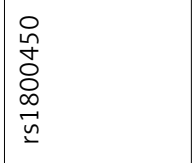 & 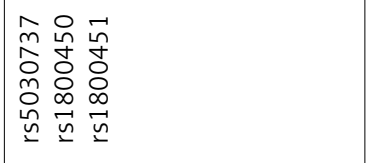 & 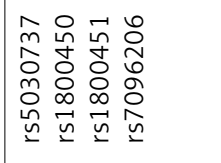 & 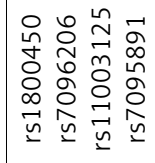 & 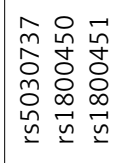 \\
\hline 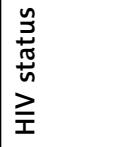 & 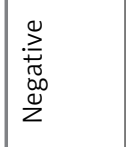 & 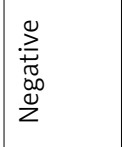 & 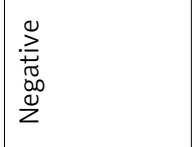 & 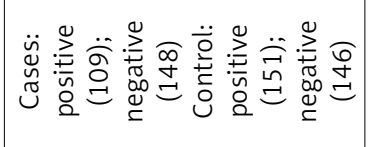 & 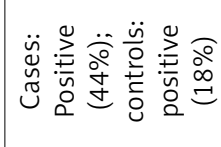 & 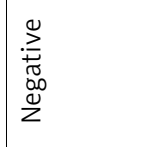 & 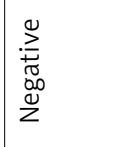 \\
\hline 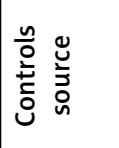 & 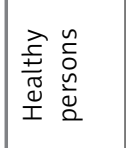 & 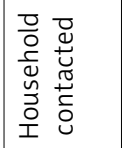 & 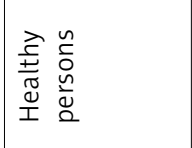 & 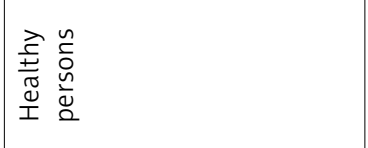 & 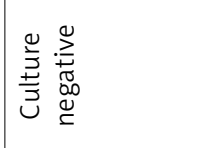 & 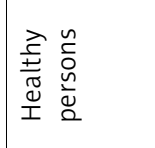 & 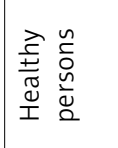 \\
\hline 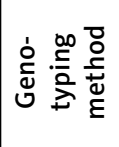 & $\begin{array}{l}\hat{u} \\
\hat{\nu} \\
\tilde{u} \\
\alpha\end{array}$ & 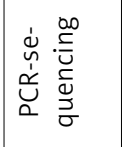 & 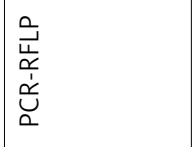 & 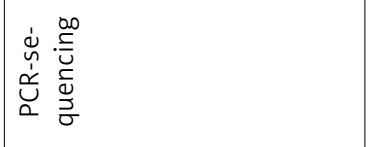 & $\begin{array}{l}\hat{u} \\
\hat{1} \\
\tilde{c} \\
\alpha\end{array}$ & 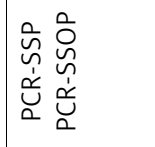 & 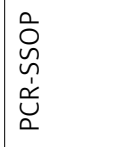 \\
\hline 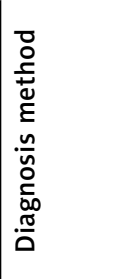 & 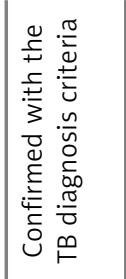 & 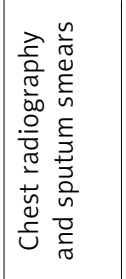 & 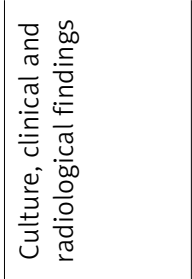 & 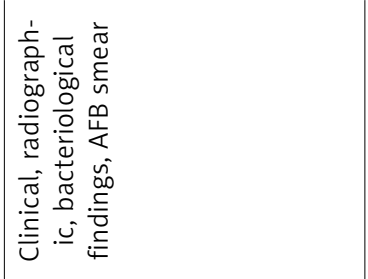 & 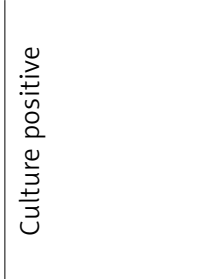 & 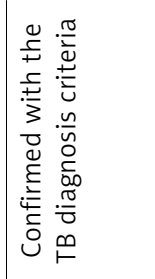 & 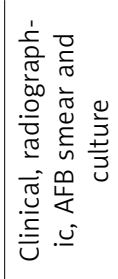 \\
\hline 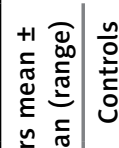 & 足 & $\begin{array}{l}7 \\
+1 \\
q \\
q\end{array}$ & $\stackrel{\infty}{\stackrel{\infty}{\sim}} \stackrel{\hat{\sigma}}{\stackrel{0}{+}+}$ & $\begin{array}{ll}\hat{\infty} & \stackrel{0}{\infty} \\
\stackrel{\infty}{\sim} & \infty \\
\dot{m} & \infty \\
+1\end{array}$ & 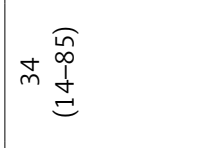 & 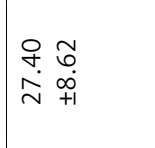 & 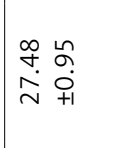 \\
\hline 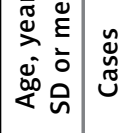 & 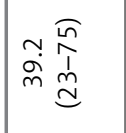 & $\begin{array}{l}\vec{y} \\
+1 \\
\dot{f}\end{array}$ & 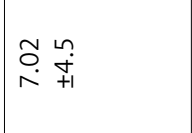 & 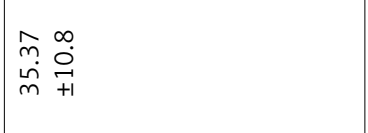 & 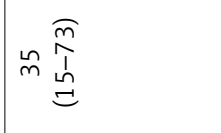 & 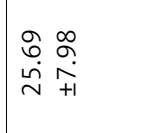 & 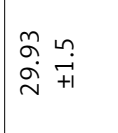 \\
\hline 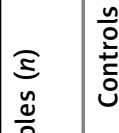 & $\underset{\sim}{\sim}$ & $\stackrel{\infty}{\infty} \underset{\sim}{\infty}$ & ন & సે & $\underset{\mathscr{f}}{\stackrel{\sim}{\sim}}$ & $\stackrel{m}{\sim}$ & $\stackrel{\infty}{+}$ \\
\hline 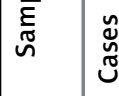 & $\overrightarrow{\tilde{v}}$ & $\stackrel{\wedge}{\sim}$ & \& & $\stackrel{\hat{n}}{\sim}$ & $\stackrel{n}{f}$ & $\stackrel{\sim}{\sim}$ & $\stackrel{\infty}{\infty}$ \\
\hline 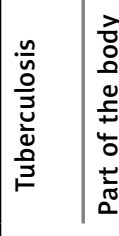 & 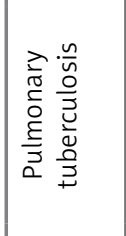 & 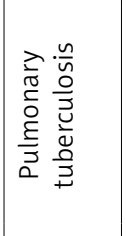 & 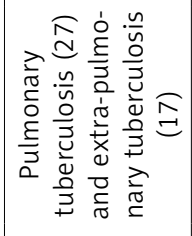 & 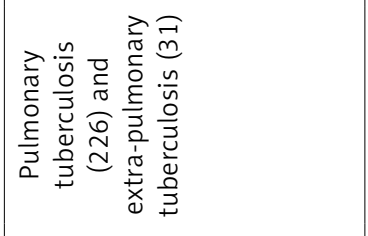 & 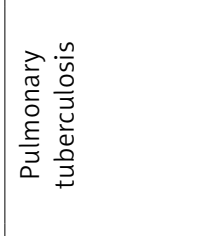 & 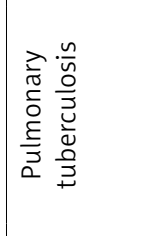 & 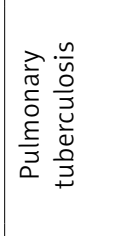 \\
\hline 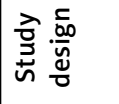 & 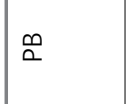 & 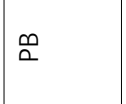 & 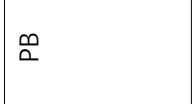 & 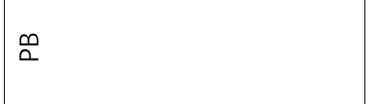 & 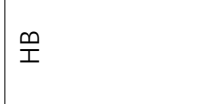 & 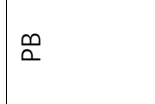 & $\dddot{\dddot{I}}$ \\
\hline 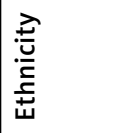 & 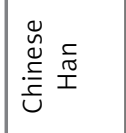 & 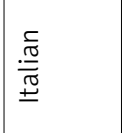 & $\frac{\frac{c}{n}}{. \frac{5}{5}}$ & 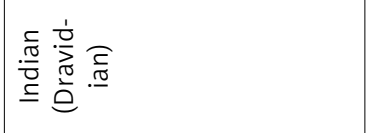 & 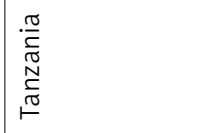 & 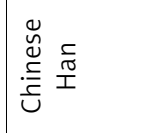 & 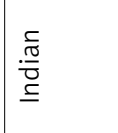 \\
\hline 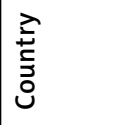 & : & $\frac{\text { }}{\underline{\oplus}}$ & 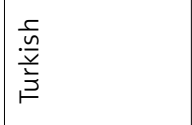 & 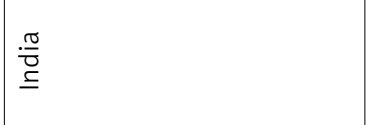 & 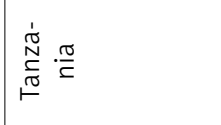 & : & 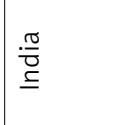 \\
\hline 蒙竞 & 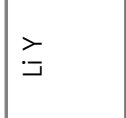 & 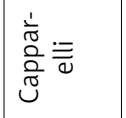 & 产 & 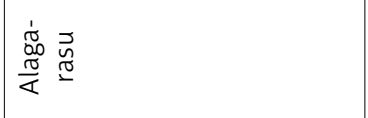 & $\begin{array}{l}\infty \\
\dot{0} \\
\circ \\
0 \\
i\end{array}$ & $\begin{array}{l}3 \\
. \unlhd \\
\exists\end{array}$ & $\begin{array}{l}\cdot \overline{0} \\
\frac{\pi}{\pi} \\
\frac{2}{2} \\
\sim\end{array}$ \\
\hline$\stackrel{\bar{d}}{\stackrel{亠}{\nu}}$ & $\vec{\sim}$ & Oे & $\stackrel{\infty}{\infty}$ & ò & ò্ & ஜ & ஜ \\
\hline
\end{tabular}




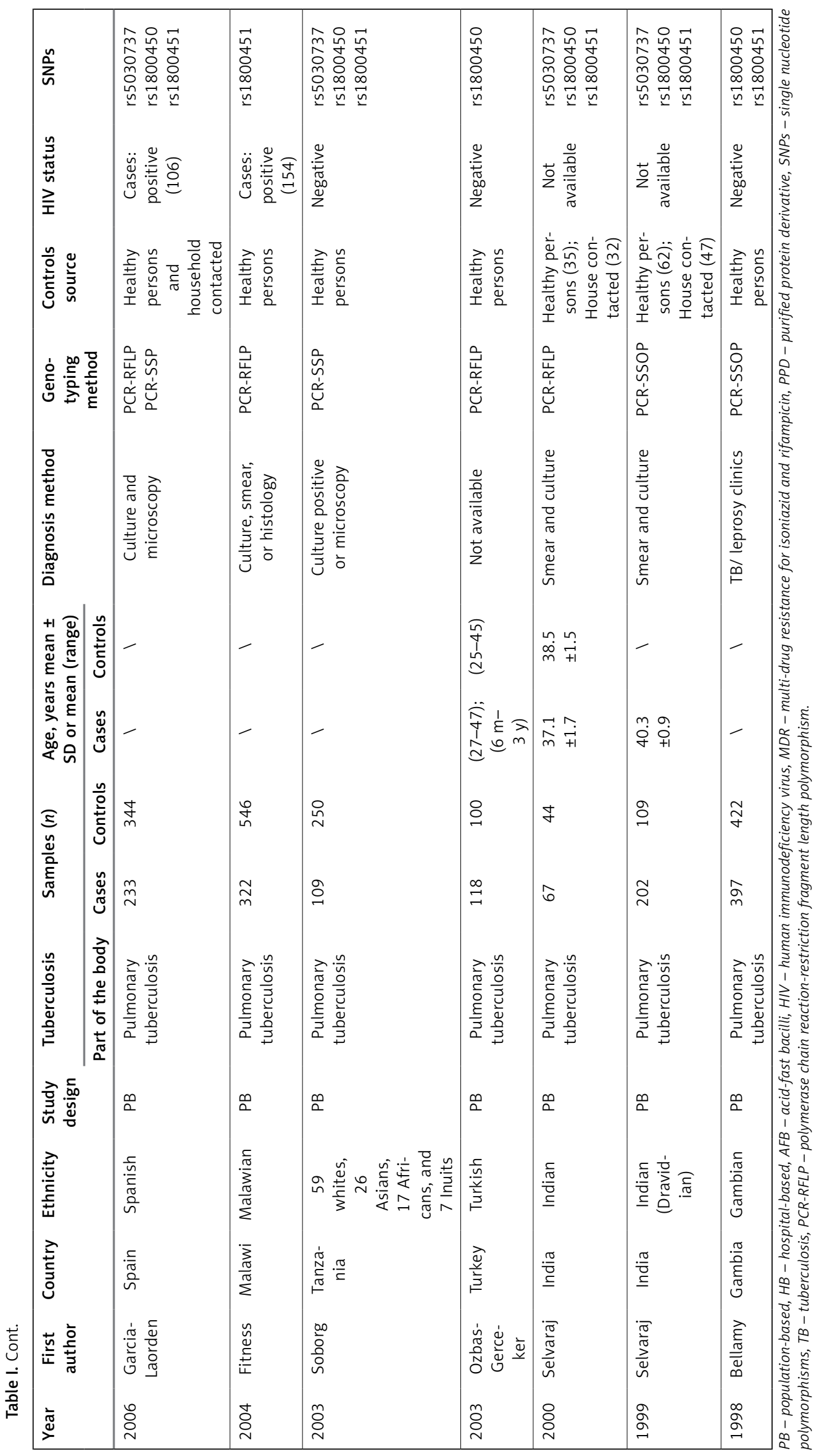


Table II. Multiple comparisons of genotype effects

\begin{tabular}{|c|c|c|c|c|}
\hline Genotype & & led OR ex & & \\
\hline & OR $(95 \% \mathrm{Cl})$ & $P$-value & $P^{a}$ & \\
\hline rs7096206 X/Y: & & & & Recessive \\
\hline YY vs. XX (OR1) & $0.964(0.689-1.347)$ & 0.829 & 0.970 & \\
\hline XY vs. XX (OR2) & $1.290(1.004-1.657)$ & 0.047 & 0.005 & \\
\hline YY vs. XY (OR3) & $0.828(0.587-1.168)$ & 0.282 & 0.739 & \\
\hline A/O T/C: & & & & Recessive \\
\hline CC vs. TT (OR1) & $1.973(0.935-4.163)$ & 0.075 & 0.000 & \\
\hline CT vs. TT (OR2) & $1.179(0.852-1.633)$ & 0.321 & 0.000 & \\
\hline CC vs. CT (OR3) & $1.547(0.954-2.507)$ & 0.077 & 0.001 & \\
\hline rs11003125 H/L: & & & & Codominant \\
\hline LL vs. HH (OR1) & $0.716(0.501-1.024)$ & 0.062 & 0.501 & \\
\hline LH vs. HH (OR2) & $0.890(0.692-1.144)$ & 0.798 & 0.052 & \\
\hline LL vs. LH (OR3) & $0.911(0.645-1.286)$ & 0.454 & 0.432 & \\
\hline rs5030737 A/D: & & & & Recessive \\
\hline DD vs. AA (OR1) & $2.985(0.712-12.510)$ & 0.135 & 0.718 & \\
\hline AD vs. AA (OR2) & $1.021(0.746-1.395)$ & 0.898 & 0.499 & \\
\hline DD vs. AD (OR3) & $3.054(0.696-13.395)$ & 0.139 & 0.510 & \\
\hline rs1800450 A/B: & & & & Dominant \\
\hline BB vs. AA (OR1) & $0.989(0.544-1.797)$ & 0.971 & 0.117 & \\
\hline AB vs. AA (OR2) & $0.911(0.717-1.157)$ & 0.443 & 0.014 & \\
\hline BB vs. AB (OR3) & $1.074(0.621-1.856)$ & 0.798 & 0.232 & \\
\hline rs1800451 A/C: & & & & Codominant \\
\hline CC vs. AA (OR1) & $0.833(0.697-0.995)$ & 0.044 & 0.709 & \\
\hline AC vs. AA (OR2) & $0.955(0.802-1.138)$ & 0.607 & 0.085 & \\
\hline CC vs. AC (OR3) & $0.894(0.747-1.070)$ & 0.222 & 0.699 & \\
\hline rs7095891 P/Q: & & & & Recessive \\
\hline QQ vs. PP (OR1) & $1.089(0.906-1.309)$ & 0.362 & 0.815 & \\
\hline PQ vs. PP (OR2) & $0.953(0.841-1.080)$ & 0.449 & 0.845 & \\
\hline QQ vs. PQ (OR3) & $1.133(0.948-1.353)$ & 0.169 & 0.882 & \\
\hline
\end{tabular}

rs 11003125 polymorphisms and the risk of TB were included in the meta-analysis. For rs11003125, the estimated OR1 (GG vs. CC), OR2 (CG vs. CC) and OR3 (GG vs. CG) were 0.716 (95\% Cl: 0.501-1.024), 0.890 (95\% Cl: $0.693-1.144)$ and 0.911 (95\% Cl: $0.645-$ 1.286) (Table II). Thus, we mainly pooled ORs for allele comparison and the codominant genetic model in the subgroup analysis by ethnicity. The pooled examination revealed no significant association between rs11003125 polymorphism and the risk of tuberculosis (G/C: $\mathrm{OR}=0.89,95 \% \mathrm{Cl}: 0.58-1.35, p=$ $0.572, p^{a}<0.001$; CG vs. CC + GG: OR $=1.00,95 \% \mathrm{Cl}$ : $\left.0.87-1.16, p=0.946, p^{a}=0.290\right)$ (Table III).

\section{MBL2 rs7095891 polymorphism}

Three case-control studies (2325 cases and 2668 controls) on the relationship between the rs7095891 polymorphism and the risk of TB were included in the meta-analysis. For rs7095891, the estimated OR1 (TT vs. CC), OR2 (CT vs. CC) and 


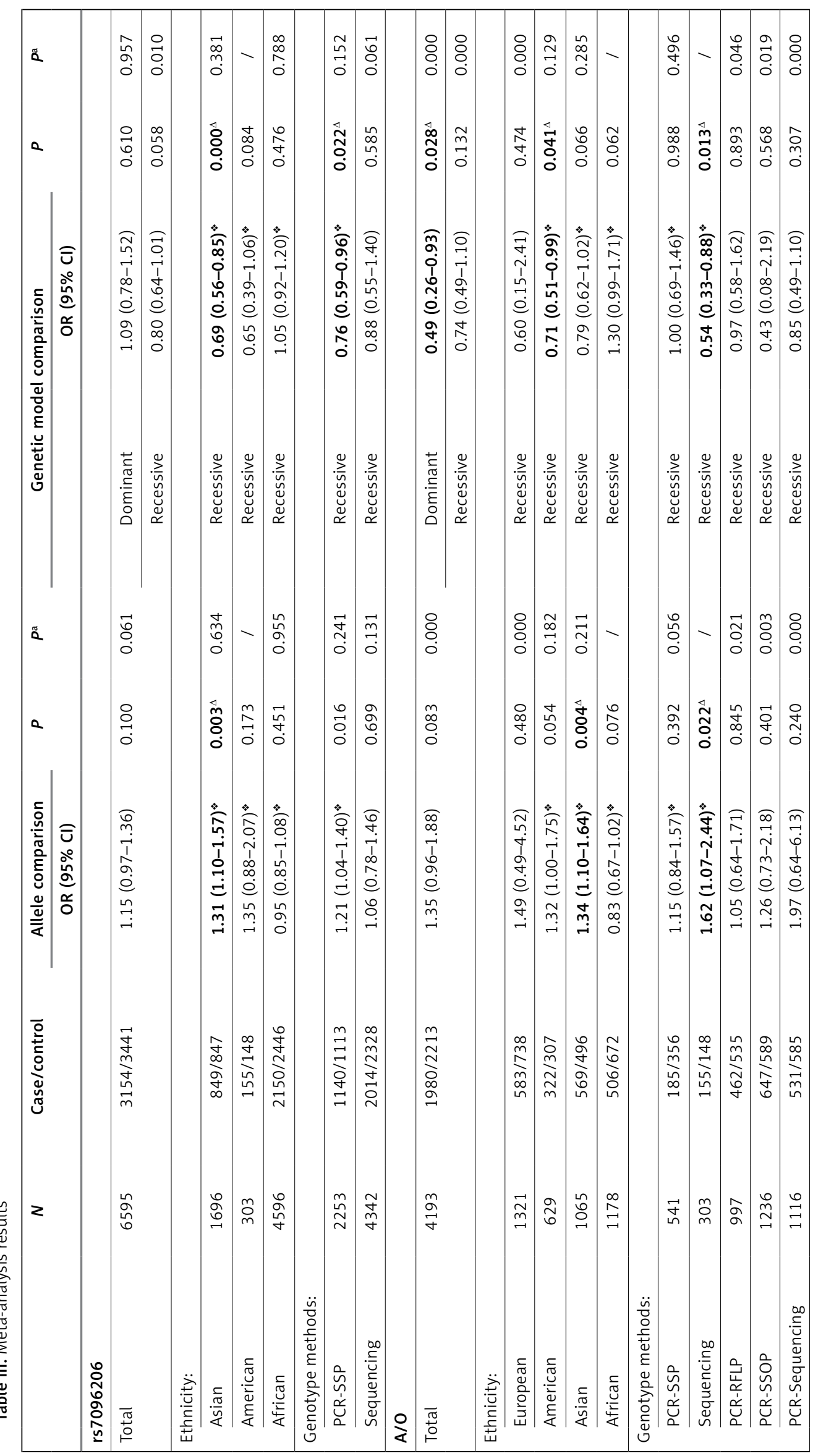




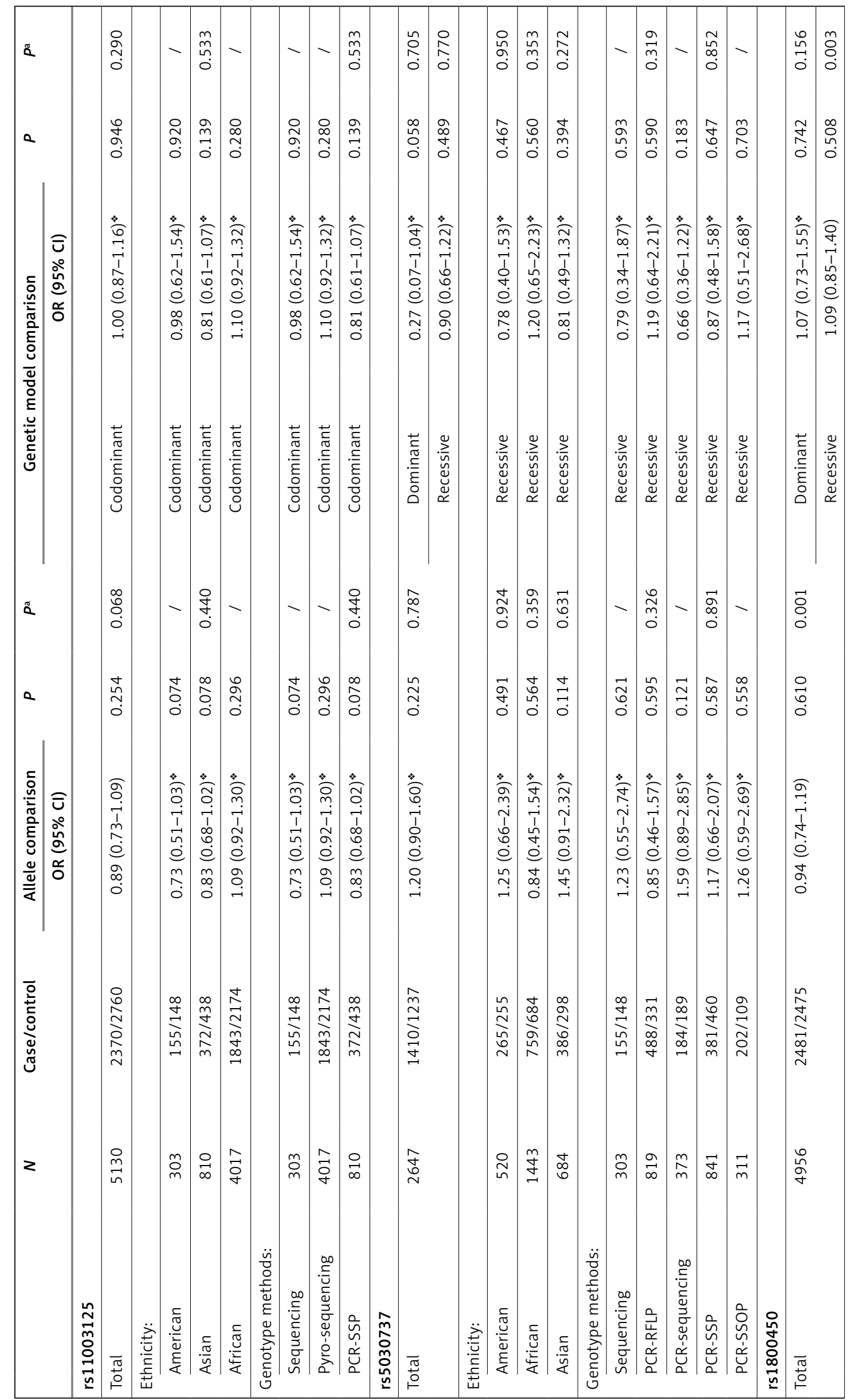




\begin{tabular}{|c|c|c|c|c|c|c|c|c|c|c|c|c|c|c|c|c|c|c|c|c|c|c|}
\hline 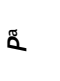 & & - & $\begin{array}{l}\hat{n} \\
\hat{o} \\
0\end{array}$ & $\begin{array}{l}\vec{v} \\
\vec{m} \\
\dot{0}\end{array} \mid$ & 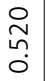 & & - & 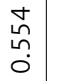 & $\begin{array}{l}8 \\
\stackrel{2}{2} \\
\vdots \\
0\end{array}$ & - & - & $\frac{m}{\bar{c}}$ & & $\begin{array}{l}\infty \\
0 \\
0 \\
0 \\
0\end{array}$ & $\mid \begin{array}{l}0 \\
0 \\
0 \\
0\end{array}$ & - & & $-\mid \begin{array}{c}\tilde{D} \\
\\
0\end{array}$ & - & - & $\begin{array}{c}\infty \\
\stackrel{1}{-} \\
0\end{array}$ & - \\
\hline$a$ & & \begin{tabular}{|l|}
$\infty$ \\
$\overrightarrow{0}$ \\
0 \\
0
\end{tabular} & $\begin{array}{l}\infty \\
0 \\
0 \\
0 \\
0\end{array}$ & $\begin{array}{l}\vec{\sigma} \\
\vec{\sigma} \\
\dot{0}\end{array}$ & $\begin{array}{l}\circ \\
\stackrel{\circ}{\circ} \\
0\end{array}$ & & $\begin{array}{l}\infty \\
\overrightarrow{0} \\
0 \\
0\end{array}$ & $\begin{array}{l}1 \tilde{n} \\
\tilde{o} \\
\dot{0}\end{array}$ & $\begin{array}{c}\stackrel{m}{n} \\
\stackrel{n}{n} \\
0\end{array}$ & 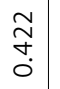 & 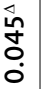 & $\begin{array}{l}\text { w } \\
\text { wू }\end{array}$ & & $\begin{array}{l}0 \\
0 \\
0 \\
0 \\
0\end{array}$ & $\begin{array}{c}\stackrel{N}{\mathcal{f}} \\
0\end{array}$ & $\begin{array}{c}\hat{\hat{m}} \\
\hat{0}\end{array}$ & & $\begin{array}{c}n \\
0 \\
0 \\
0\end{array}$ & $\mid \begin{array}{l}\hat{\sigma} \\
\hat{\sigma} \\
0\end{array}$ & $\begin{array}{c}\hat{n} \\
\tilde{m} \\
0\end{array}$ & $\begin{array}{l}\tilde{n} \\
\hat{n} \\
0\end{array}$ & $\begin{array}{l}\stackrel{0}{\circ} \\
\stackrel{\leftrightarrow}{0}\end{array}$ \\
\hline 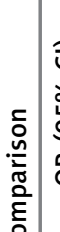 & & 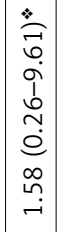 & 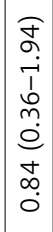 & 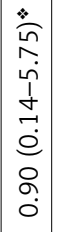 & 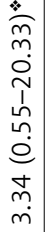 & & 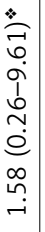 & 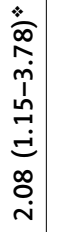 & 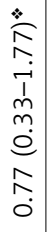 & 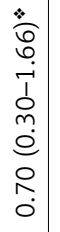 & 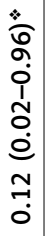 & 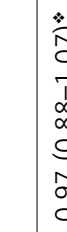 & & 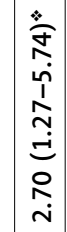 & 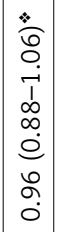 & 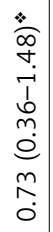 & & 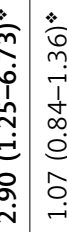 & 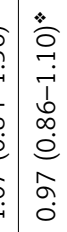 & 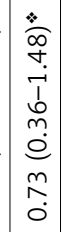 & 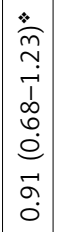 & 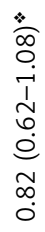 \\
\hline 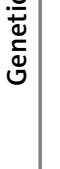 & & 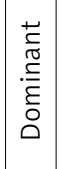 & 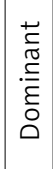 & 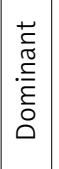 & 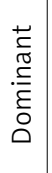 & & 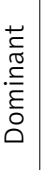 & 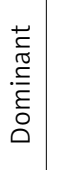 & 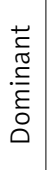 & 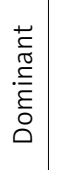 & 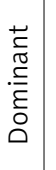 & 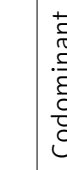 & & 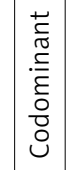 & 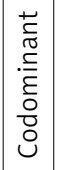 & 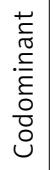 & & 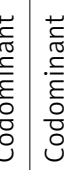 & 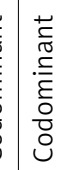 & 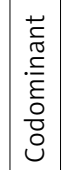 & 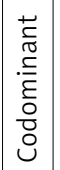 & 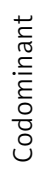 \\
\hline ฉू & & - & $\begin{array}{l}\overrightarrow{8} \\
0\end{array}$ & $\begin{array}{l}\tilde{N} \\
\hat{\sim} \\
0\end{array}$ & $\begin{array}{c}\infty \\
m \\
m \\
0\end{array}$ & & - & $\underset{\underset{N}{\sim}}{\stackrel{ \pm}{0}}$ & $\begin{array}{l}\stackrel{n}{2} \\
\stackrel{n}{0} \\
0\end{array}$ & - & 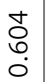 & $\frac{3}{2}$ & & $\begin{array}{l}\hat{N} \\
\hat{0}\end{array}$ & $\begin{array}{l}0 \\
0 \\
0 \\
0\end{array}$ & - & - & 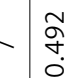 & - & - & $\begin{array}{l}8 \\
0 \\
0 \\
0\end{array}$ & - \\
\hline$a$ & & $\begin{array}{c}a \\
\hat{n} \\
0\end{array}$ & 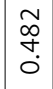 & $\left|\begin{array}{c}0 \\
\stackrel{0}{0} \\
0\end{array}\right|$ & $\begin{array}{l}\overrightarrow{8} \\
\dot{0} \\
\dot{0}\end{array}$ & & 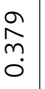 & $\begin{array}{l}\overrightarrow{8} \\
\dot{0}\end{array}$ & $\begin{array}{l}0 \\
\infty \\
\stackrel{0}{0} \\
0\end{array}$ & $\begin{array}{l}\stackrel{n}{\hat{f}} \\
\dot{f} \\
0\end{array}$ & $\begin{array}{l}0 \\
0 \\
0 \\
0 \\
0\end{array}$ & 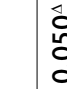 & & $\begin{array}{l}\underset{\Xi}{\tilde{O}} \\
\stackrel{0}{0}\end{array}$ & $\mid \begin{array}{l}\hat{1} \\
\tilde{o} \\
0 \\
\dot{0}\end{array}$ & $\begin{array}{l}\infty \\
\infty \\
\sim \\
0\end{array}$ & & $\begin{array}{c}0 \\
0 \\
0 \\
0 \\
0\end{array}$ & 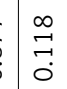 & $\mid \begin{array}{c}\infty \\
\infty \\
\sim \\
0\end{array}$ & $\mid \begin{array}{l}\vec{b} \\
0 \\
0\end{array}$ & $\begin{array}{l}\vec{m} \\
\stackrel{0}{0} \\
\dot{0}\end{array}$ \\
\hline 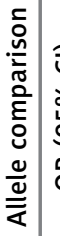 & & 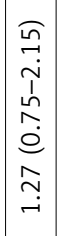 & 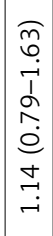 & 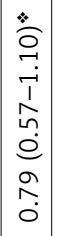 & 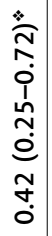 & & 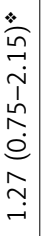 & $\begin{array}{c}\dot{0} \\
\hat{0} \\
0 \\
0 \\
1 \\
n \\
0 \\
0 \\
o \\
0 \\
0\end{array}$ & 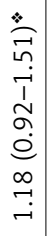 & 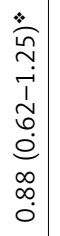 & 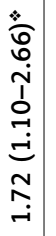 & 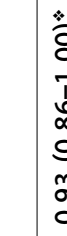 & & 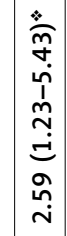 & 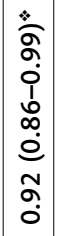 & 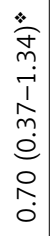 & & 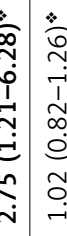 & 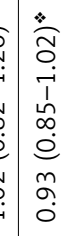 & 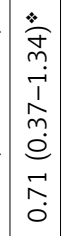 & 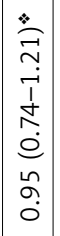 & 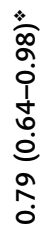 \\
\hline 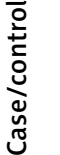 & & $\begin{array}{l}\infty \\
\stackrel{\infty}{\sim} \\
\stackrel{\omega}{n} \\
\sim\end{array}$ & 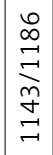 & 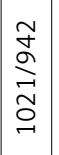 & 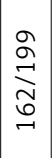 & & 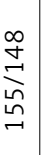 & 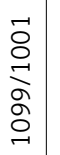 & 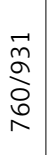 & 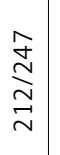 & 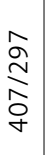 & 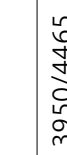 & & $\begin{array}{l}\stackrel{\vec{n}}{\stackrel{D}{ }} \\
\stackrel{\sim}{N}\end{array}$ & $\mid \begin{array}{l}\tilde{N} \\
0 \\
\infty \\
\infty \\
\tilde{m} \\
m\end{array}$ & 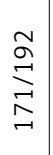 & 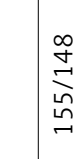 & 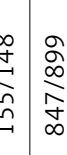 & $\mid \begin{array}{l}\infty \\
\tilde{N} \\
\text { N } \\
\tilde{m} \\
\infty \\
\sim\end{array}$ & 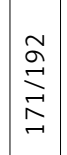 & $\mid \begin{array}{c}n \\
\hat{n} \\
\infty \\
o \\
\sigma\end{array}$ & 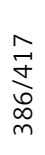 \\
\hline$z$ & & $\begin{array}{l}m \\
\tilde{m}\end{array}$ & $\underset{\sim}{\stackrel{\sim}{\sim}}$ & $\mid \begin{array}{l}m \\
\stackrel{2}{\sigma} \\
\sigma\end{array}$ & $\overrightarrow{\overrightarrow{0}}$ & & ్ְ & $\begin{array}{l}\stackrel{\otimes}{\vec{N}} \\
\underset{\sim}{2}\end{array}$ & $\begin{array}{l}\vec{\sigma} \\
\hat{\sigma}\end{array}$ & $\begin{array}{l}\text { ô } \\
\forall\end{array}$ & $\hat{m}$ & $\stackrel{n}{\vec{\alpha}}$ & & $\approx$ & \begin{tabular}{l}
0 \\
\multirow{1}{n}{} \\
\end{tabular} & $\begin{array}{l}m \\
\dot{n} \\
n\end{array}$ & $\stackrel{m}{m}$ & $\begin{array}{l}n \\
n \\
n\end{array}$ & $\underset{\forall}{\stackrel{\Im}{\forall}}$ & $\begin{array}{l}\hat{n} \\
\tilde{m}\end{array}$ & $\overrightarrow{\overrightarrow{0}}$ & $\underset{\infty}{m}$ \\
\hline & 孚 & 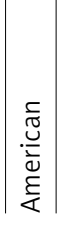 & $\frac{\pi}{4}$ & 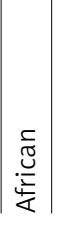 & 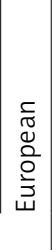 & 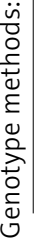 & 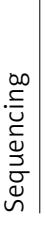 & 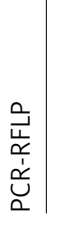 & $\begin{array}{l}\hat{n} \\
\hat{\alpha} \\
\tilde{u} \\
\alpha\end{array}$ & 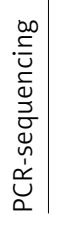 & 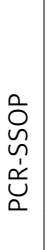 & 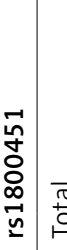 & 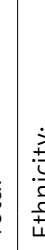 & 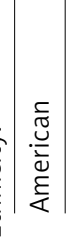 & 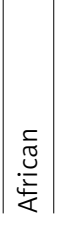 & $\frac{c}{\frac{c}{\pi}}$ & 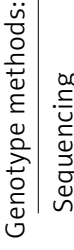 & 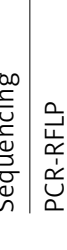 & 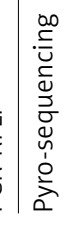 & 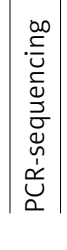 & $\mid$ & 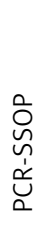 \\
\hline
\end{tabular}




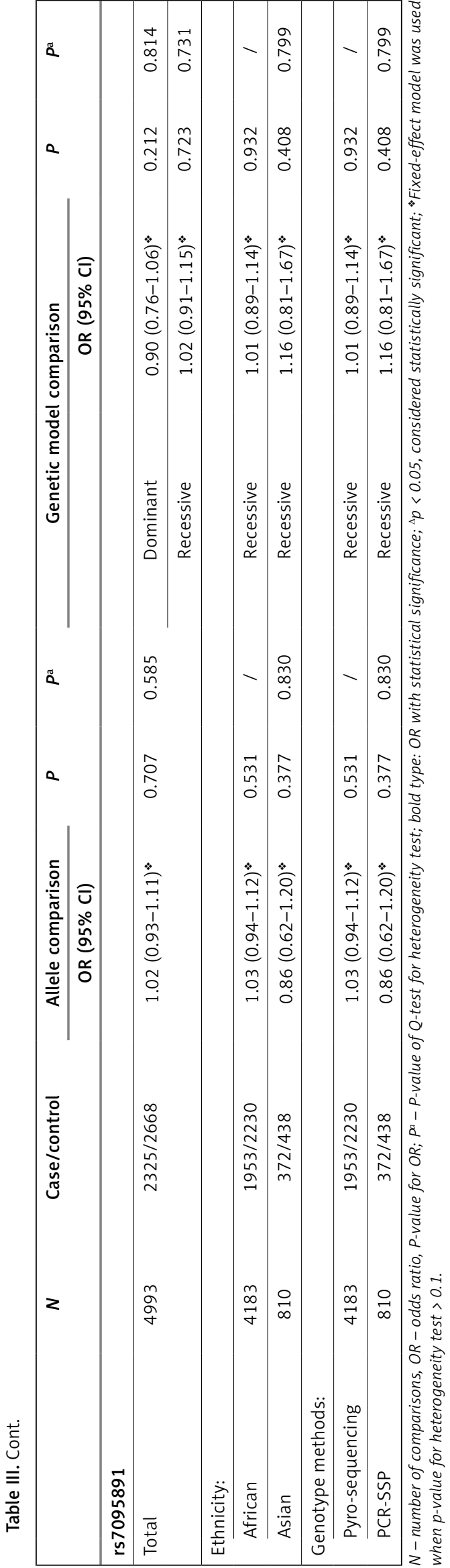

OR3 (TT vs. CT) were 1.089 (95\% Cl: 0.906-1.309), 0.953 (95\% Cl: $0.841-1.080)$ and $1.133(95 \% \mathrm{Cl}$ : 0.948-1.353) (Table II). Thus, we mainly pooled ORs for allele comparison and the recessive genetic model in the subgroup analysis by ethnicity. The pooled examination revealed no significant association between rs7095891 polymorphism and the risk of tuberculosis ( $\mathrm{T} / \mathrm{C}: \mathrm{OR}=1.02$, 95\% Cl: 0.93-1.11, $p=0.707, p^{a}=0.585$; CC vs. $\mathrm{CT}+\mathrm{TT}: \mathrm{OR}=1.02,95 \% \mathrm{Cl}: 0.91-1.15, p=0.723$, $\left.p^{a}=0.731\right)$ (Table III).

\section{MBL2 rs5030737 polymorphism}

Seven case-control studies (1410 cases and 1237 controls) on the relationship between the rs5030737 polymorphism and the risk of TB were included in the meta-analysis. For rs5030737, the estimated OR1 (DD versus AA), OR2 (AD vs. $A A)$ and OR3 (DD vs. AD) were $2.985(95 \% \mathrm{Cl}$ : $0.712-12.51), 1.021$ (95\% Cl: 0.746-1.395) and 3.054 (95\% Cl: 0.696-13.40) (Table II). Thus, we mainly pooled ORs for allele comparison and the recessive genetic model in the subgroup analysis by ethnicity. The pooled examination revealed no significant association between rs5030737 polymorphism and the risk of tuberculosis (D/A: OR $=1.20,95 \% \mathrm{Cl}: 0.90-1.60, p=0.225, p^{a}=0.787$; $A A$ vs. $A D+D D: O R=0.90,95 \% C l: 0.66-1.22, p=$ $0.489, p^{a}=0.770$ ) (Table III).

\section{MBL2 rs1800450 polymorphism}

Thirteen case-control studies contained sufficient data for analysis of the relationship between the rs1800450 polymorphism and the risk of TB. The distribution of genotypes from the study of Mauro et al. was not consistent with HWE (Table IV), so only twelve studies (2481 cases and 2493 controls) were included in the meta-analysis [20]. For rs1800450, the estimated OR1 (BB vs. AA), OR2 ( $A B$ vs. $A A$ ) and OR3 (BB vs. $A B$ ) were 0.989 (95\% Cl: 0.544-1.797), 0.911 (95\% Cl: 0.717-1.157) and 1.074 (95\% Cl: 0.621-1.856) (Table II). Thus, we mainly pooled ORs for allele comparison and the dominant genetic model in the subgroup analysis by ethnicity. The pooled examination revealed no significant association between rs 1800450 polymorphism and the risk of tuberculosis $(B / A: O R=0.94$, 95\% Cl: 0.74-1.19, $p=0.610, p^{a}=0.001 ; \mathrm{AA}+\mathrm{AB}$ vs. $\mathrm{BB}: \mathrm{OR}=0.83,95 \% \mathrm{Cl}: 0.62-1.10, p=0.742$, $\left.p^{a}=0.156\right)$ (Table III). When stratified by ethnicity, a significantly decreased risk was found among Europeans in allele contrast (B/A: $\mathrm{OR}=0.42,95 \% \mathrm{Cl}$ : $0.25-0.72, p=0.001, p^{a}=0.338$ ) (Table III, Figure 3). When stratified by genotyping method, the allele comparison (B/A: $\mathrm{OR}=0.68,95 \% \mathrm{Cl}: 0.57-0.83$, $\left.p<0.001, p^{a}=0.224\right)$ and dominant genetic model (AA + AB vs. BB: $\mathrm{OR}=2.08,95 \% \mathrm{Cl}: 1.15-3.78, p=$ $\left.0.015, p^{\mathrm{a}}=0.554\right)$ remained statistically significant 
Table IV. Distribution of gene polymorphisms of studies included in the meta-analysis

\begin{tabular}{|c|c|c|c|c|c|c|c|c|c|c|c|c|c|c|}
\hline \multirow[t]{2}{*}{ SNP } & \multirow[t]{2}{*}{ Year } & \multirow{2}{*}{$\begin{array}{l}\text { First } \\
\text { author }\end{array}$} & \multicolumn{5}{|c|}{ Cases } & \multicolumn{5}{|c|}{ Controls } & \multicolumn{2}{|c|}{ HWE } \\
\hline & & & & & & & & & & & & & $\chi^{2}$ & $P$-value \\
\hline \multirow[t]{7}{*}{ rs7096206 } & & & YY & $\mathrm{YX}$ & $x x$ & $Y$ & $x$ & YY & YX & $X X$ & $\mathrm{Y}$ & $x$ & & \\
\hline & 2015 & Chen & 325 & 166 & 12 & 816 & 190 & 296 & 113 & 10 & 705 & 133 & 0.0411 & 0.8393 \\
\hline & 2014 & Chen & 123 & 77 & 5 & 323 & 87 & 159 & 49 & 8 & 367 & 65 & 2.7405 & 0.0978 \\
\hline & 2013 & da Cruz & 101 & 49 & 5 & 251 & 59 & 110 & 32 & 6 & 252 & 44 & 3.1437 & 0.0762 \\
\hline & 2011 & Thye & 1437 & 396 & 26 & 3270 & 448 & 1663 & 486 & 31 & 3812 & 548 & 0.4491 & 0.5028 \\
\hline & 2007 & Soborg & 182 & 96 & 13 & 460 & 122 & 166 & 85 & 15 & 417 & 115 & 0.8652 & 0.3523 \\
\hline & 2006 & Liu W & 91 & 44 & 6 & 226 & 56 & 151 & 54 & 7 & 356 & 68 & 0.6227 & 0.4300 \\
\hline \multirow[t]{13}{*}{$\mathrm{A} / \mathrm{O}$} & & & AA & AO & 00 & A & 0 & AA & AO & 00 & A & 0 & & \\
\hline & 2014 & $\begin{array}{l}\text { Garcia- } \\
\text { Gasalla }\end{array}$ & 48 & 24 & 4 & 120 & 32 & 71 & 34 & 1 & 176 & 36 & 2.0077 & 0.1565 \\
\hline & 2013 & da Cruz & 92 & 55 & 8 & 239 & 71 & 108 & 34 & 6 & 250 & 46 & 2.3077 & 0.1287 \\
\hline & 2013 & Araujo & 102 & 62 & 3 & 266 & 68 & 101 & 56 & 2 & 258 & 60 & 3.5960 & 0.0579 \\
\hline & 2009 & $\begin{array}{l}\text { Cappar- } \\
\text { elli }\end{array}$ & 55 & 158 & 61 & 268 & 280 & 166 & 112 & 10 & 444 & 132 & 2.9226 & 0.0873 \\
\hline & 2008 & $\begin{array}{c}\text { Alaga- } \\
\text { rasu }\end{array}$ & 145 & 87 & 25 & 377 & 137 & 169 & 109 & 19 & 447 & 147 & 0.0638 & 0.8006 \\
\hline & 2007 & Soborg & 289 & 132 & 22 & 710 & 176 & 271 & 131 & 30 & 673 & 191 & 6.1675 & 0.0130 \\
\hline & 2006 & Selvaraj & 24 & 19 & 5 & 67 & 29 & 37 & 18 & 3 & 92 & 24 & 0.1713 & 0.6789 \\
\hline & 2006 & $\begin{array}{l}\text { Garcia- } \\
\text { Laorden }\end{array}$ & 144 & 79 & 10 & 367 & 99 & 183 & 134 & 27 & 500 & 188 & 0.1273 & 0.7213 \\
\hline & 2003 & Soborg & 71 & 30 & 8 & 172 & 46 & 157 & 86 & 7 & 400 & 100 & 1.4063 & 0.2357 \\
\hline & 2000 & Selvaraj & 32 & 24 & 6 & 88 & 36 & 22 & 9 & 1 & 53 & 11 & 0.0046 & 0.9458 \\
\hline & 1999 & Selvaraj & 107 & 73 & 22 & 287 & 117 & 68 & 39 & 2 & 175 & 43 & 1.8374 & 0.1753 \\
\hline & 1998 & Bellamy & 198 & 166 & 33 & 562 & 232 & 183 & 197 & 42 & 563 & 281 & 1.0967 & 0.2950 \\
\hline \multirow[t]{5}{*}{ rs11003125 } & & & LL & $\mathrm{HL}$ & $\mathrm{HH}$ & $\mathbf{L}$ & $\mathrm{H}$ & LL & $\mathrm{HL}$ & $\mathrm{HH}$ & L & $\mathrm{H}$ & & \\
\hline & 2013 & da Cruz & 82 & 63 & 10 & 227 & 83 & 68 & 61 & 19 & 197 & 99 & 0.8147 & 0.3667 \\
\hline & 2011 & Thye & 1570 & 265 & 8 & 3405 & 281 & 1878 & 287 & 9 & 4043 & 305 & 0.3115 & 0.5768 \\
\hline & 2011 & Li Y & 105 & 92 & 34 & 302 & 160 & 89 & 106 & 31 & 284 & 168 & 0.0040 & 0.9498 \\
\hline & 2006 & Liu W & 44 & 66 & 31 & 154 & 128 & 49 & 105 & 58 & 203 & 221 & 0.0124 & 0.9114 \\
\hline \multirow[t]{8}{*}{ rs5030737 } & & & AA & $A D$ & DD & A & D & AA & $A D$ & DD & A & D & & \\
\hline & 2013 & da Cruz & 142 & 12 & 1 & 296 & 14 & 138 & 9 & 1 & 285 & 11 & 3.3406 & 0.0676 \\
\hline & 2013 & Araujo & 102 & 8 & 0 & 212 & 8 & 101 & 6 & 0 & 208 & 6 & 0.0890 & 0.7654 \\
\hline & 2011 & de Wit & 363 & 15 & 0 & 741 & 15 & 211 & 13 & 0 & 435 & 13 & 0.2000 & 0.6547 \\
\hline & 2008 & $\begin{array}{c}\text { Alaga- } \\
\text { rasu }\end{array}$ & 156 & 26 & 2 & 338 & 30 & 169 & 20 & 0 & 358 & 20 & 0.5899 & 0.4425 \\
\hline & 2007 & Soborg & 289 & 8 & 0 & 586 & 8 & 271 & 6 & 0 & 548 & 6 & 0.0332 & 0.8554 \\
\hline & 2003 & Soborg & 71 & 12 & 1 & 154 & 14 & 157 & 25 & 1 & 339 & 27 & 0.0001 & 0.9965 \\
\hline & 1999 & Selvaraj & 186 & 9 & 7 & 381 & 23 & 99 & 10 & 0 & 208 & 10 & 0.2519 & 0.6157 \\
\hline
\end{tabular}


Table IV. Cont.

\begin{tabular}{|c|c|c|c|c|c|c|c|c|c|c|c|c|c|c|}
\hline \multirow[t]{2}{*}{ SNP } & \multirow[t]{2}{*}{ Year } & \multirow{2}{*}{$\begin{array}{c}\text { First } \\
\text { author }\end{array}$} & \multicolumn{5}{|c|}{ Cases } & \multicolumn{5}{|c|}{ Controls } & \multicolumn{2}{|c|}{ HWE } \\
\hline & & & & & & & & & & & & & $\chi^{2}$ & $P$-value \\
\hline \multirow[t]{14}{*}{ rs1800450 } & & & AA & $A B$ & BB & A & B & $A A$ & $A B$ & BB & A & B & & \\
\hline & 2013 & da Cruz & 122 & 31 & 2 & 275 & 35 & 124 & 21 & 3 & 269 & 27 & 3.0756 & 0.0795 \\
\hline & 2013 & Araujo & 102 & 50 & 0 & 254 & 50 & 101 & 48 & 0 & 250 & 48 & 5.4927 & 0.0191 \\
\hline & 2011 & Singla & 218 & 126 & 13 & 562 & 152 & 207 & 155 & 30 & 569 & 215 & 0.0174 & 0.8951 \\
\hline & 2011 & de Wit & 363 & 63 & 2 & 789 & 67 & 211 & 50 & 0 & 472 & 50 & 2.9288 & 0.0870 \\
\hline & 2011 & $\mathrm{LiY}$ & 171 & 57 & 3 & 399 & 63 & 186 & 37 & 3 & 409 & 43 & 0.5442 & 0.4607 \\
\hline & 2008 & Cosar & 40 & 4 & 0 & 84 & 4 & 71 & 27 & 1 & 169 & 29 & 0.8162 & 0.3663 \\
\hline & 2008 & $\begin{array}{c}\text { Alaga- } \\
\text { rasu }\end{array}$ & 156 & 44 & 12 & 356 & 68 & 169 & 68 & 10 & 406 & 88 & 0.8828 & 0.3474 \\
\hline & 2007 & Soborg & 289 & 9 & 0 & 587 & 9 & 271 & 13 & 1 & 555 & 15 & 3.4429 & 0.0635 \\
\hline & 2006 & Liu W & 103 & 34 & 4 & 240 & 42 & 166 & 42 & 4 & 374 & 50 & 0.4824 & 0.4873 \\
\hline & 2003 & Soborg & 71 & 16 & 3 & 158 & 22 & 157 & 48 & 3 & 362 & 54 & 0.0960 & 0.7567 \\
\hline & 2003 & $\begin{array}{l}\text { Ozbas- } \\
\text { Gerce- } \\
\text { ker }\end{array}$ & 101 & 16 & 1 & 218 & 18 & 76 & 20 & 4 & 172 & 28 & 2.8708 & 0.0902 \\
\hline & 1999 & Selvaraj & 137 & 51 & 14 & 325 & 79 & 84 & 24 & 1 & 192 & 26 & 0.2519 & 0.6157 \\
\hline & 1998 & Bellamy & 198 & 7 & 0 & 403 & 7 & 183 & 5 & 0 & 371 & 5 & 0.0341 & 0.8534 \\
\hline \multirow[t]{11}{*}{ rs1800451 } & & & AA & $\mathrm{AC}$ & $\mathrm{CC}$ & $A$ & C & $\mathrm{AA}$ & $A C$ & $\mathrm{CC}$ & A & C & & \\
\hline & 2013 & da Cruz & 133 & 22 & 0 & 288 & 22 & 140 & 8 & 0 & 288 & 8 & 0.1142 & 0.7354 \\
\hline & 2013 & Araujo & 102 & 4 & 0 & 208 & 4 & 101 & 2 & 0 & 204 & 2 & 0.0099 & 0.9207 \\
\hline & 2011 & Thye & 885 & 815 & 193 & 2585 & 1201 & 1002 & 977 & 257 & 2981 & 1491 & 0.6457 & 0.4217 \\
\hline & 2011 & de Wit & 363 & 56 & 0 & 782 & 56 & 211 & 39 & 0 & 461 & 39 & 1.7892 & 0.1810 \\
\hline & 2008 & $\begin{array}{c}\text { Alaga- } \\
\text { rasu }\end{array}$ & 156 & 14 & 1 & 326 & 16 & 169 & 21 & 2 & 359 & 25 & 1.9782 & 0.1596 \\
\hline & 2007 & Soborg & 289 & 115 & 20 & 693 & 155 & 271 & 112 & 20 & 654 & 152 & 3.4039 & 0.0650 \\
\hline & 2004 & Fitness & 205 & 105 & 12 & 515 & 129 & 362 & 160 & 24 & 884 & 208 & 1.3527 & 0.2448 \\
\hline & 2003 & Soborg & 71 & 2 & 1 & 144 & 4 & 157 & 13 & 0 & 327 & 13 & 0.2687 & 0.6042 \\
\hline & 1999 & Selvaraj & 176 & 25 & 1 & 377 & 27 & 103 & 5 & 1 & 211 & 7 & 7.4832 & 0.0062 \\
\hline & 1998 & Bellamy & 198 & 159 & 29 & 555 & 217 & 183 & 192 & 42 & 558 & 276 & 0.6585 & 0.4171 \\
\hline \multirow[t]{4}{*}{ rs7095891 } & & & PP & $P Q$ & $\mathrm{QQ}$ & $\mathbf{P}$ & Q & PP & $P Q$ & QQ & $\mathbf{P}$ & Q & & \\
\hline & 2011 & Thye & 725 & 920 & 308 & 2370 & 1536 & 825 & 1086 & 319 & 2736 & 1724 & 1.6089 & 0.2046 \\
\hline & 2011 & Li Y & 189 & 39 & 3 & 417 & 45 & 181 & 41 & 4 & 403 & 49 & 0.8556 & 0.3550 \\
\hline & 2006 & Liu W & 118 & 22 & 1 & 258 & 24 & 171 & 39 & 2 & 381 & 43 & 0.0185 & 0.8918 \\
\hline
\end{tabular}

HWE - Hardy-Weinberg equilibrium.

in PCR-RFLP studies. We also found a significant association of TB in polymerase chain reaction sequence-specific oligonucleotide probe (PCR-SSOP) studies for two comparison models: the allele model (B/A: OR $=1.72,95 \% \mathrm{Cl}: 1.10-2.66, p=0.016, p^{2}$ $=0.604)$ and the dominant model (AA $+A B$ vs. BB: $\mathrm{OR}=0.12,95 \% \mathrm{Cl}: 0.02-0.96, p=0.045$ ) (Table III).

\section{MBL2 rs1800451 polymorphism}

Excluding the study of Selvaraj et al., which was not consistent with HWE (Table IV) [36], nine case-control studies (3950 cases and 4465 controls) on the relationship between the $\mathrm{rs} 1800451$ polymorphism and the risk of TB were included in the meta-analysis. For rs 1800451 , the estimated 
A

Study

OR $(95 \% \mathrm{Cl}) \quad$ Weight (\%)

ID

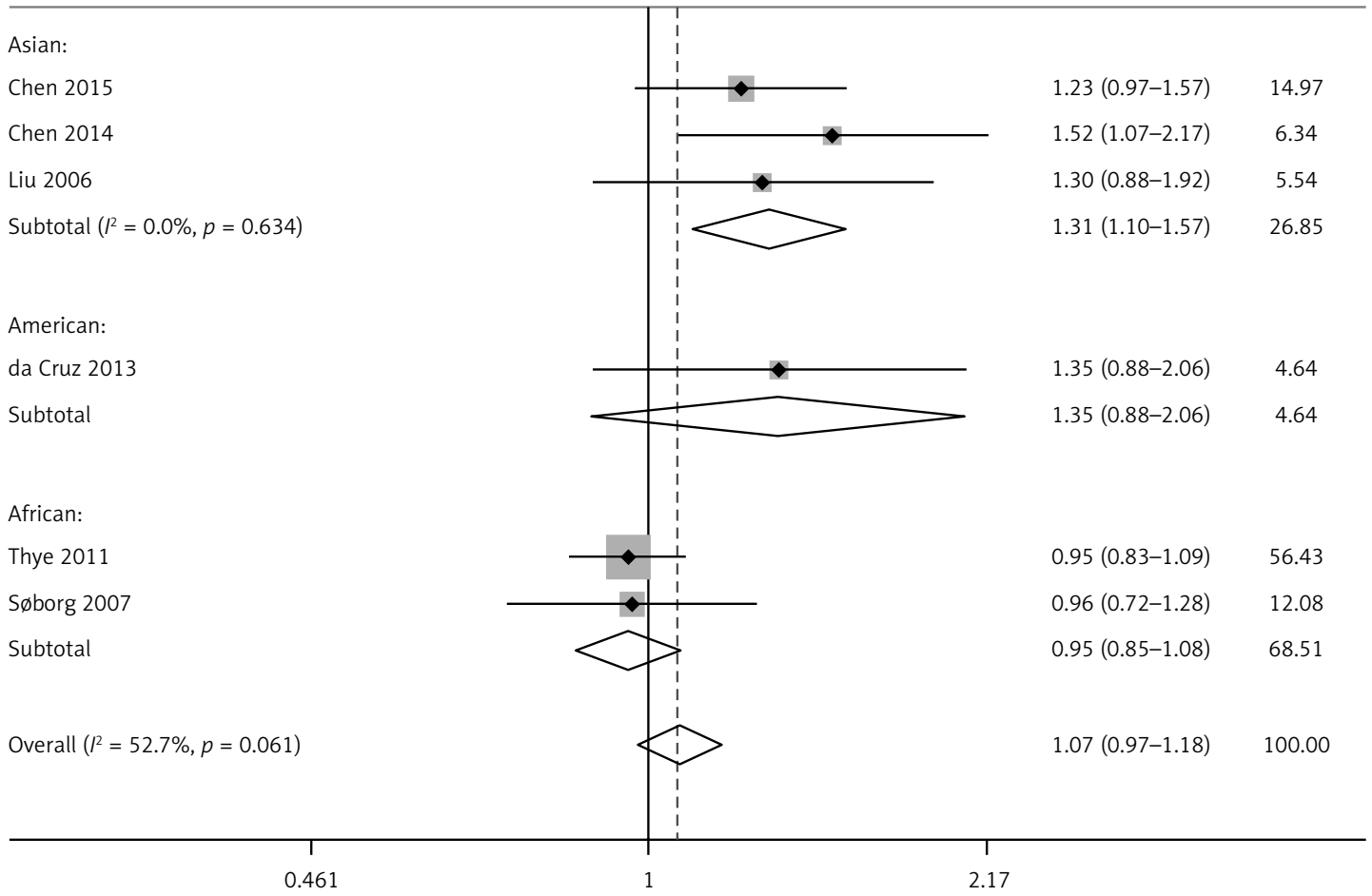

B

Study

OR $(95 \% \mathrm{Cl}) \quad$ Weight $(\%)$

ID

Asian:

Chen 2015

Chen 2014

Liu 2006

Subtotal $\left(I^{2}=0.0 \%, p=0.381\right)$

American:

da Cruz 2013

Subtotal

African:

Thye 2011

Søborg 2007

Subtotal $\left(I^{2}=0.0 \%, p=0.788\right)$

Overall $\left(P^{2}=66.7 \%, p=0.010\right)$

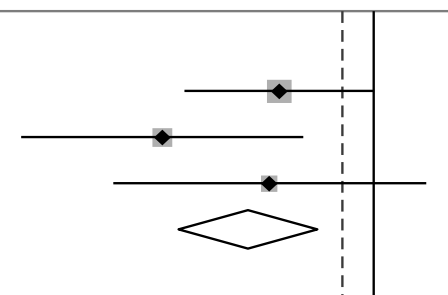

$0.76(0.57-1.00) \quad 17.04$

$0.54(0.36-0.81) \quad 9.24$

$0.74(0.47-1.16) \quad 6.38$

$0.69(0.56-0.85) \quad 32.65$

$0.65(0.39-1.06) \quad 5.85$

$0.65(0.39-1.06) \quad 5.85$

$1.06(0.91-1.23) \quad 51.81$

$1.01(0.71-1.42) \quad 9.69$

$1.05(0.92-1.20) \quad 61.50$

$0.91(0.82-1.01) \quad 100.00$

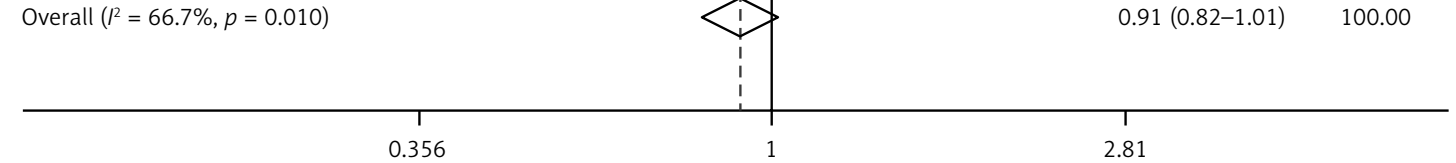

Figure 2. Forest plot of tuberculosis risk associated with MBL2 rs7096206 polymorphism (A - allele comparison: C allele vs. G allele. B - recessive comparison: GG vs. GC + CC) 


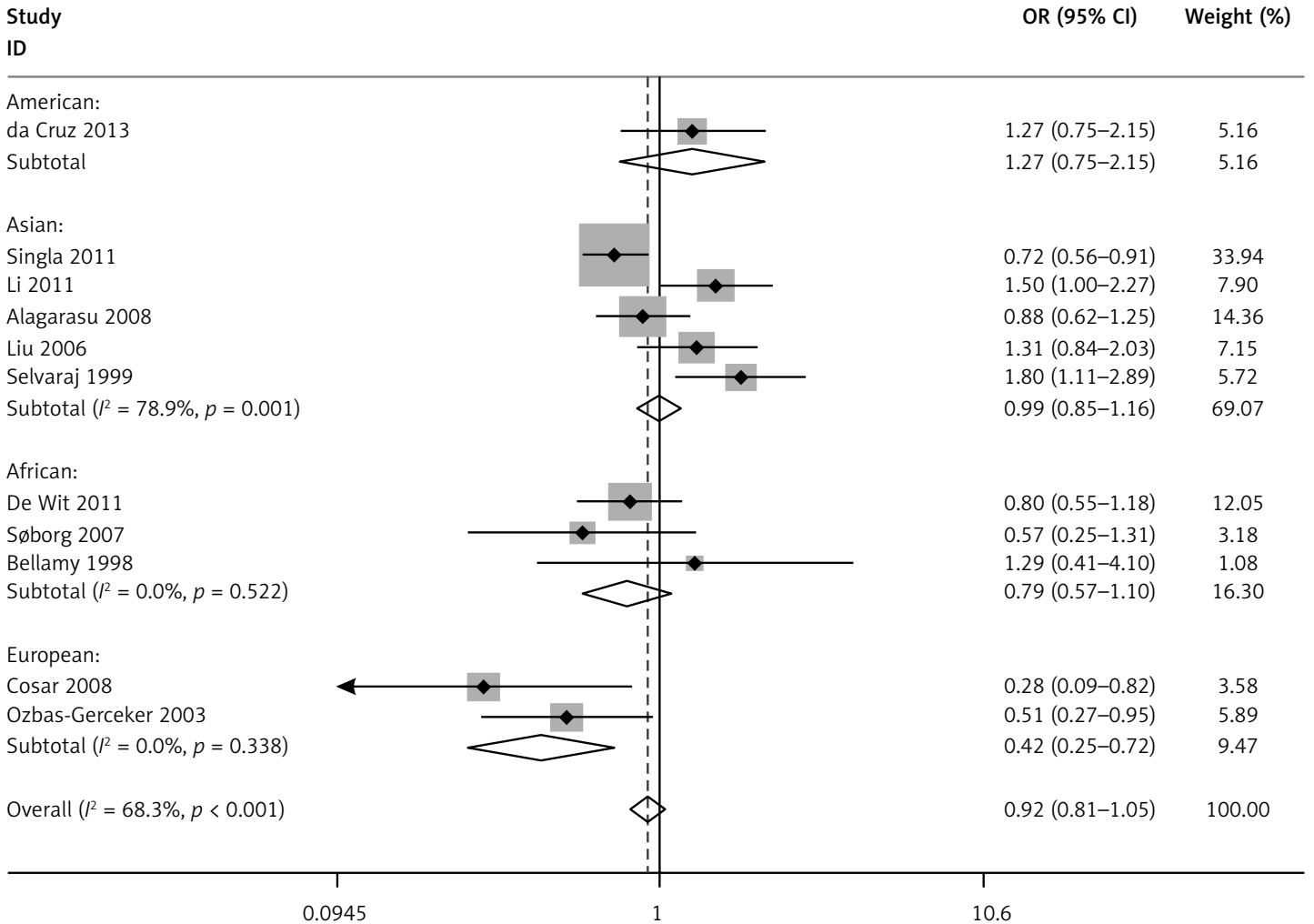

Figure 3. Forest plot of tuberculosis risk associated with MBL2 rs1800450 polymorphism (allele comparison: $B$ allele vs. A allele)

OR1 (CC vs. AA), OR2 (AC vs. AA) and OR3 (CC vs. $A C)$ were $0.833(95 \% \mathrm{Cl}$ : 0.697-0.995), 0.955 (95\% Cl: 0.802-1.138) and 0.894 (95\% Cl: 0.7471.070) (Table II). Thus, we mainly pooled ORs for allele comparison and the codominant genetic model in the subgroup analysis by ethnicity. The pooled examination revealed a significant association between rs1800451 polymorphism and the risk of tuberculosis ( $\mathrm{C} / \mathrm{A}: \mathrm{OR}=0.93,95 \% \mathrm{Cl}$ : 0.86-1.00, $p=0.050, p^{a}=0.152$ ) (Table III). When performing a meta-analysis by ethnicity, increased risk of TB was found among Americans $(\mathrm{C} / \mathrm{A}: \mathrm{OR}=2.59,95 \% \mathrm{Cl}: 1.23-5.43, p=0.012$, $p^{a}=0.727 ; A C$ vs. $A A+C C: O R=2.70,95 \% \mathrm{Cl}:$ 1.27-5.74, $\left.p=0.010, p^{a}=0.698\right)$, and a protective effect was observed among Africans (C/A: $\mathrm{OR}=0.92,95 \% \mathrm{Cl}: 0.86-0.99, p=0.035, p^{a}=$ 0.460) (Table III, Figure 4). For the subgroup analysis by genotyping method, the allele comparison (C/A: OR $=2.75,95 \% \mathrm{Cl}: 1.21-6.28, p=0.016)$ and recessive genetic model (AC vs. AA + CC: OR $=2.90,95 \% \mathrm{Cl}: 1.25-6.73, p=0.013)$ remained statistically significant in sequencing studies. We also found a decreased risk of TB in PCRSSOP studies in the allele model ( $C / A: O R=0.79$, 95\% Cl: 0.64-0.98, $p=0.031$ ) (Table III).

\section{MBL2 exon 1 polymorphisms}

Excluding the study of Søborg et al., which was not consistent with HWE (Table IV) [28], elev- en case-control studies (1980 cases and 2213 controls) on the relationship between the MBL2 exon 1 polymorphisms (wild-type (AA) versus any $M B L 2$ variant allele (OA/OO) genotype) and the risk of TB were included in the meta-analysis. The estimated OR1 (OO vs. AA), OR2 (AO vs. AA) and OR3 (OO vs. AO) were 1.973 (95\% Cl: 0.935-4.163), 1.179 (95\% Cl: $0.852-1.633)$ and $1.547(95 \% \mathrm{Cl}$ : 0.954-2.507) (Table II). Thus, we mainly pooled OR for allele comparison and the recessive genetic model in the subgroup analysis by ethnicity. Overall, a significant association between exon 1 gene polymorphisms and the risk of TB was observed (AA + AO vs. OO: $O R=0.49,95 \% \mathrm{Cl}: 0.26-0.93$, $\left.p=0.028, p^{a}=0.000\right)$ (Table III). The results of subgroup analysis based on ethnicity indicated that MBL2 O allele carriers ( $\mathrm{OO}$ and/or OO) in Asian populations were associated with increased risk of TB (O/A: OR $=1.34,95 \% \mathrm{Cl}: 1.10-1.64, p=0.004$, $\left.p^{a}=0.211\right)$, and a significant protective effect was detected between $M B L 2$ exon 1 polymorphisms and TB risk in Americans under recessive models (AA vs. $\mathrm{AO}+\mathrm{OO}: \mathrm{OR}=0.71,95 \% \mathrm{Cl}: 0.51-0.99$, $\left.p=0.041, p^{a}=0.129\right)$, suggesting genetic diversity among ethnicities (Table III, Figure 5). For the subgroup analysis by genotyping method, the allele comparison $(\mathrm{O} / \mathrm{A}: \mathrm{OR}=1.62,95 \% \mathrm{Cl}$ : $1.07-2.44$, $p=0.022$ ) and recessive genetic model (AA vs. AO $+\mathrm{OO}: \mathrm{OR}=0.54,95 \% \mathrm{Cl}: 0.33-0.88, p=0.013)$ 
A

Study

OR $(95 \% \mathrm{Cl}) \quad$ Weight (\%)

ID

American:

da Cruz 2013

Araujo 2013

Subtotal $\left(R^{2}=0.0 \%, p=0.727\right)$

African:

Thye 2011

de Wit 2011

Søborg 2007

Fitness 2004

Bellamy 1998

Subtotal $\left(I^{2}=0.0 \%, p=0.460\right)$

Asian:

Alagarasu 2008

Subtotal

Overall $\left(I^{2}=33.2 \%, p=0.152\right)$

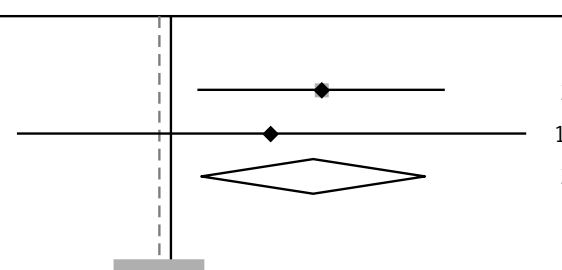

$2.75(1.20-6.28) \quad 0.52$

$1.96(0.36-10.83) \quad 0.14$

$2.59(1.23-5.43) \quad 0.66$

$0.93(0.85-1.02) \quad 63.92$

$0.85(0.55-1.29) \quad 3.12$

$0.96(0.75-1.23) \quad 8.72$

$1.06(0.83-1.36) \quad 8.45$

$0.79(0.64-0.98) \quad 13.06$

$0.92(0.86-0.99) \quad 97.28$

$0.70(0.37-1.34) \quad 1.54$

$0.70(0.37-1.34) \quad 1.54$

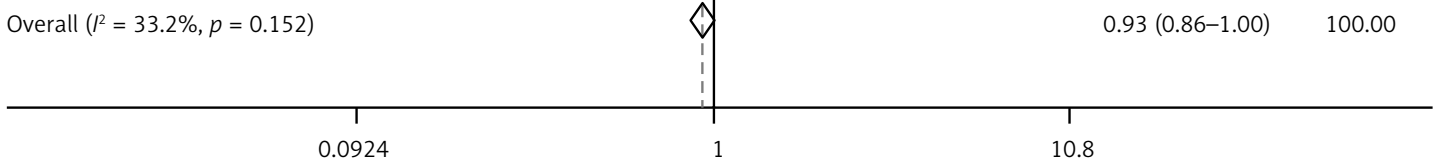

B

Study

OR $(95 \% \mathrm{Cl}) \quad$ Weight (\%)

ID

American:

da Cruz 2013

Araujo 2013

Subtotal $\left(I^{2}=0.0 \%, p=0.698\right)$

African:

Thye 2011

de Wit 2011

Søborg 2007

Fitness 2004

Bellamy 1998

Subtotal $\left(R^{2}=0.0 \%, p=0.509\right)$

Asian:

Alagarasu 2008

Subtotal

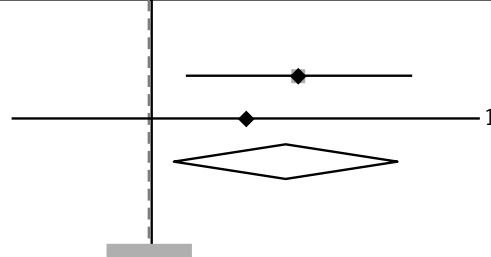

$2.89(1.25-6.73) \quad 0.82$

$1.98(0.35-11.05) \quad 0.23$

$2.70(1.27-5.74) \quad 1.04$

Overall $\left(R^{2}=38.3 \%, p=0.113\right)$

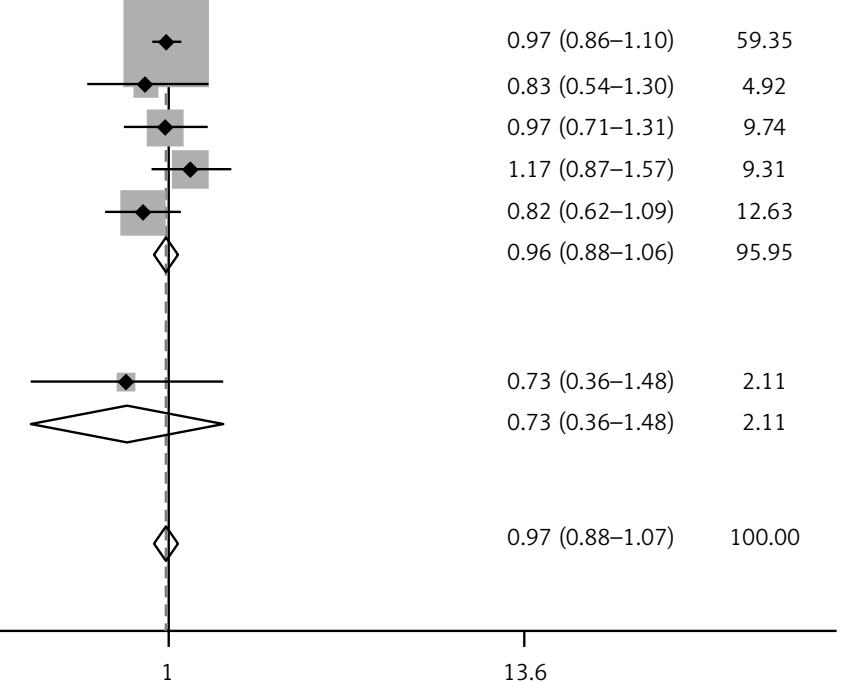

0.0738

13.6

Figure 4. Forest plot of tuberculosis risk associated with MBL2 rs1800451 polymorphism (A - allele comparison:

$C$ allele vs. A allele. $B$ - codominant comparison: $A C$ vs. $A A+C C$ ) 
A

Study

OR $(95 \% \mathrm{Cl}) \quad$ Weight (\%)

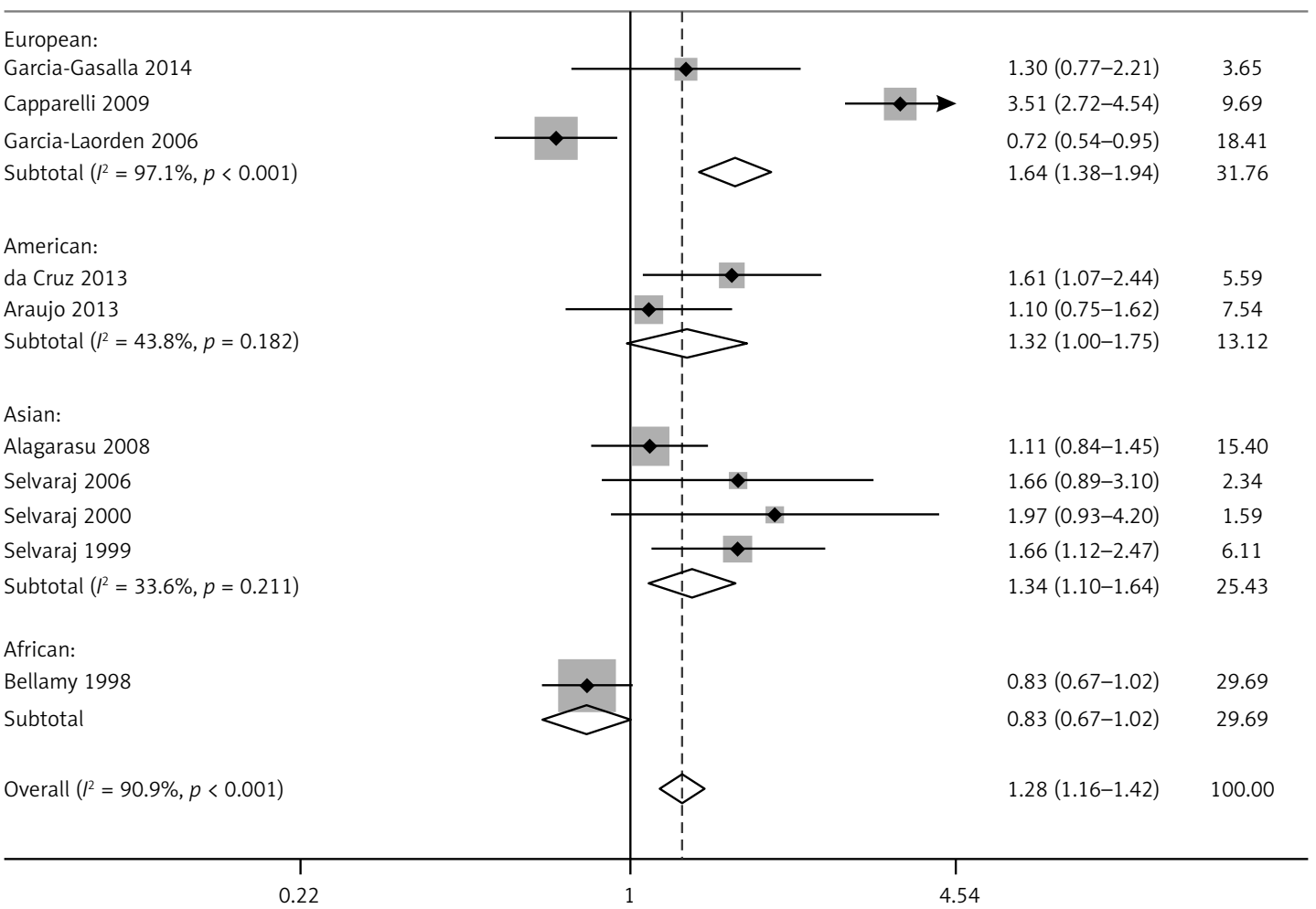

B

Study

OR $(95 \% \mathrm{Cl}) \quad$ Weight (\%)

ID

European:

Garcia-Gasalla 2014

Capparelli 2009

Garcia-Laorden 2006

Subtotal $\left(R^{2}=96.9 \%, p<0.001\right)$

American:

da Cruz 2013

Araujo 2013

Subtotal $\left(I^{2}=56.5 \%, p=0.129\right)$

Asian:

Alagarasu 2008

Selvaraj 2006

Selvaraj 2000

Selvaraj 1999

Subtotal $\left(I^{2}=20.9 \%, p=0.285\right)$

African:

Bellamy 1998

Subtotal

Overall $\left(R^{2}=89.8 \%, p<0.001\right)$
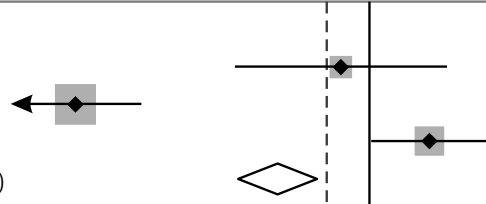

$0.85(0.46-1.57)$

$0.18(0.13-0.27)$

$1.42(1.01-2.00)$

$0.59(0.47-0.74)$

24.76

10.81

39.75

$0.54(0.33-0.88) \quad 8.60$

$0.90(0.58-1.41) \quad 7.71$

$0.71(0.51-0.99) \quad 16.30$

$0.98(0.70-1.37) \quad 13.08$

$0.57(0.26-1.24) \quad 3.21$

$0.48(0.20-1.19) \quad 2.69$

$0.68(0.42-1.09) \quad 7.95$

$0.79(0.62-1.02) \quad 26.92$

$1.30(0.99-1.71) \quad 17.02$

$1.30(0.99-1.71) \quad 17.02$

$0.79(0.69-0.89) \quad 100.00$

Figure 5. Forest plot of tuberculosis risk associated with MBL2 exon 1 polymorphisms. A - Comparison of the MBL2 exon 1 polymorphisms allele comparison (O allele vs. A allele) with tuberculosis risk. B - Comparison of the MBL2 exon 1 polymorphisms recessive comparison (AA vs. $A O+O O)$ with tuberculosis risk 
remained statistically significant in sequencing studies (Table III).

\section{Sensitivity analysis}

Sensitivity analysis was performed to evaluate the root of heterogeneity by sequentially excluding individual studies. Statistically similar results were obtained for the allele model of rs5030737, rs1800450, rs1800451, MBL2 A/O, rs11003125, rs7096206 and rs7095891 by excluding studies one after another. This indicates that this meta-analysis is stable and reliable in nature.

\section{Publication bias}

The publication bias of included studies was assessed by Begg's funnel plot and Egger's test. The funnel plots did not reveal any evidence of obvious asymmetry under the allele model (A/O, $p=0.161 ; \mathrm{rs} 1800450, p=0.732 ; \mathrm{rs} 1800451$, $p=0.754$; rs5030737, $p=0.764$; rs7095891, $p=0.296 ;$ rs11003125, $p=0.462$; rs7096206, $p=0.452$ ), and Egger's test also did not show any statistically significant evidence of publication bias under the allele model (A/O, $p=0.547$; rs1800450, $p=0.946 ; r s 1800451, p=0.538$; rs5030737, $p=0.682 ;$ rs7095891, $p=0.051$; rs11003125, $p=0.109 ;$ rs7096206, $p=0.049$ ), which indicated low risk of publication bias in this meta-analysis (Figure 6).

\section{Discussion}

The outcome of TB is modulated by the environment as well as Mycobacterium tuberculosis and hosts. Many investigations have confirmed that the genes for host susceptibility to disease appear to play the critical roles in the development of TB. MBL2 is an innate immune protein and plays a critical role in tuberculosis infection, which is elevated in active tuberculosis infection as part of an acute-phase reaction [8]. Several polymorphisms of the MBL2 gene have been identified, six of which are known for their functional effect (rs1800450, rs5030737, rs1800451, rs11003125, rs7096206 and rs709589). A number of studies have been performed to investigate the impact of $M B L 2$ gene polymorphism on susceptibility to TB in different regions and among different races. However, the clinical studies have yielded inconsistent results. To investigate these controversial issues further, we performed a comprehensive meta-analysis on the correlation between the $M B L 2$ polymorphisms and tuberculosis risk.

Based on a meta-analysis of 12 studies, Denholm et al. found no statistically significant association between $M B L 2$ genotype and pulmonary TB infection [38]. Our meta-analysis, which involved 22 studies including 7056 cases and 7764 controls, showed that the MBL2 rs7096206 and A/O polymorphisms were risk factors of TB in Asian, but not in European or African populations. Because the included participants of this meta-analysis mainly came from China and India, the results may be applicable only to East Asians. Therefore, people from East Asia who carry the MBL2 rs7096206 and $\mathrm{A} / \mathrm{O}$ gene polymorphisms may have a $31 \%$ and $34 \%$ increased TB risk, respectively.

Interestingly, the total results showed that MBL2 rs1800451 polymorphism was a protective factor, which means that persons who carry the MBL2 rs1800451 gene polymorphism may have a $7 \%$ decreased TB risk compared with the control group. In contrast, the subgroup analysis indicated that the MBL2 rs1800451 polymorphism might increase TB risk in Americans, but not in Asians or Africans. The contradiction between the overall result and subgroup result may reflect the small number of included participants belonging to the American group. Hence, more well-designed studies are required, focusing on more ethnicities to confirm the results in the future. Unfortunately, the present results suggest no significant association between the MBL2 rs5030737, rs11003125 and rs7095891 gene polymorphisms and TB risk.

Some limitations of this meta-analysis should be considered. First, some detailed information, such as age, HIV status, and types of TB (pulmonary TB and extra-pulmonary TB), was not all available, which limited our further assessment by performing stratified analysis based on those confounding factors. Secondly, some SNPs such as rs7095891 contained only 3 studies in this systematic analysis. The limited number of studies and small sample sizes restricted the power of the study. Thirdly, the significant between-study heterogeneity detected in some comparisons, different patient populations and different sources of controls may contribute to the heterogeneity. Fourthly, three studies deviated from $\operatorname{HWE}[20,28$, 36], making the sample a poor representation. We therefore conducted the meta-analyses after exclusion of these studies. However, this exclusion did not materially affect the results. Fifthly, our study could not assess gene-gene and gene-environment interactions due to the limited information of included studies. Finally, the small sample sizes in some subgroup analyses may have limited statistical power to estimate the possible risk for MBL2 polymorphisms. Only two articles on Americans were included, so we must be careful when we refer to the result. Thus, more studies are needed to confirm the association between $M B L 2$ and tuberculosis risk, especially in different ethnic populations.

In conclusion, our meta-analysis suggested that MBL2 rs7096206 and A/O gene polymorphisms may be risk factors contributing to TB 
A

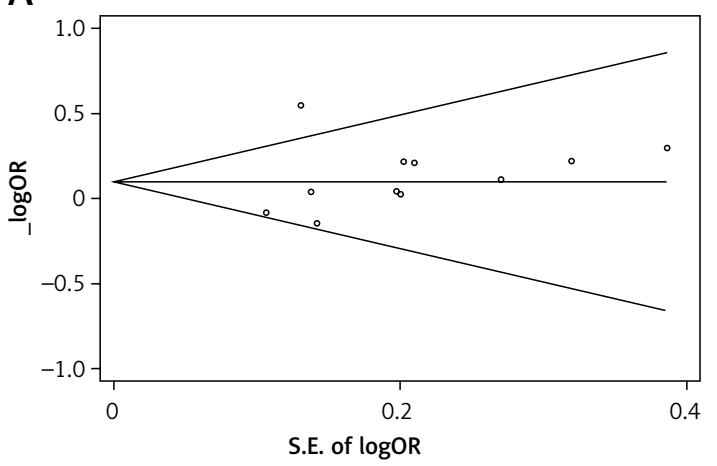

C

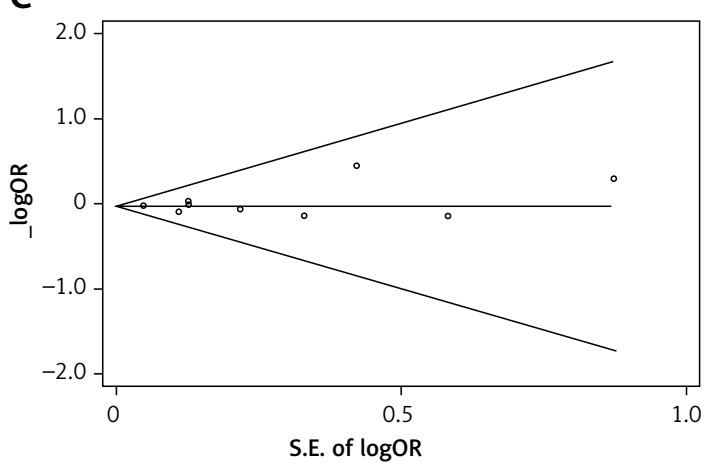

E

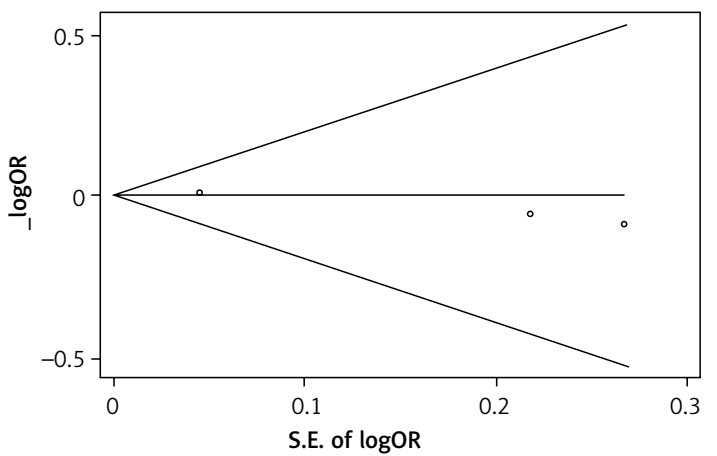

G

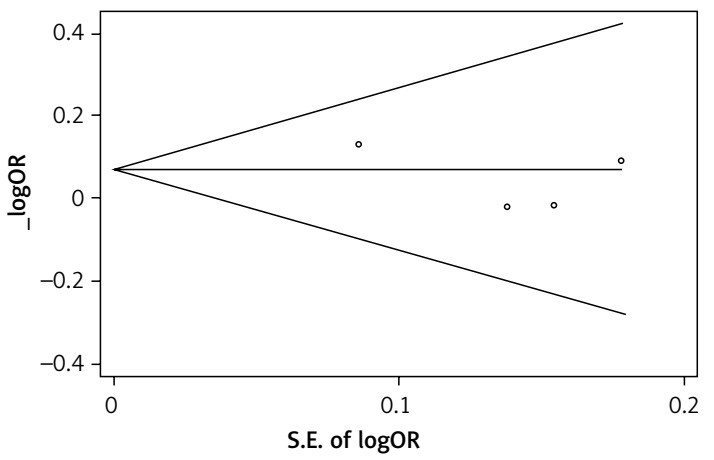

susceptibility, especially in East Asia. However, the MBL2 rs1800451 gene polymorphism may be a protective factor for TB risk. The findings of our study could be pooled in a future meta-analysis of multiple studies, providing more power to detect an association. It is critical that larger scale and well-designed epidemiological studies
B

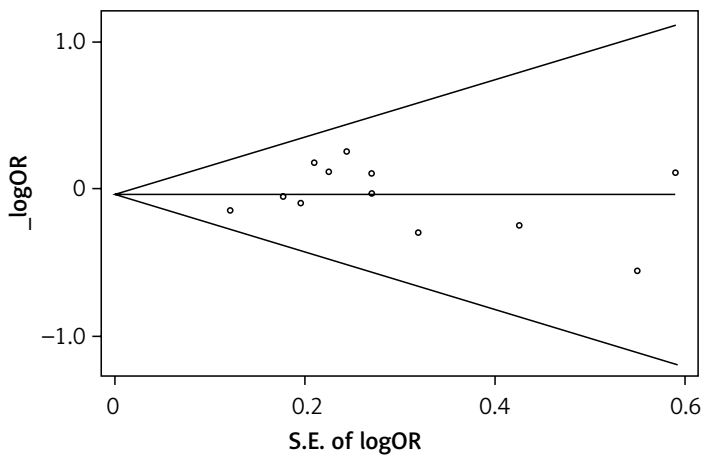

D

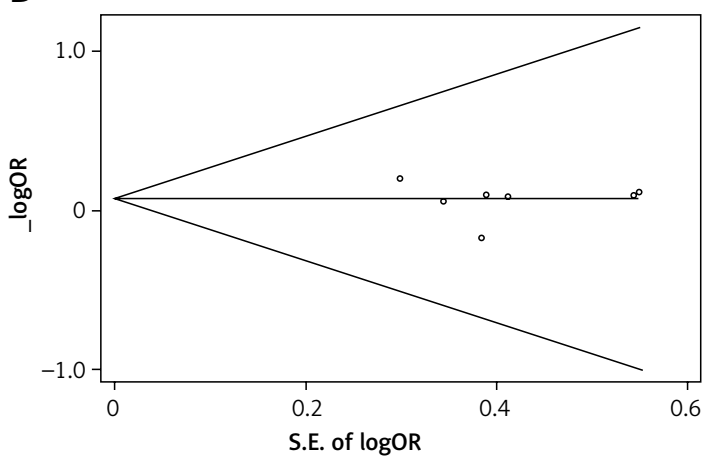

$\mathbf{F}$

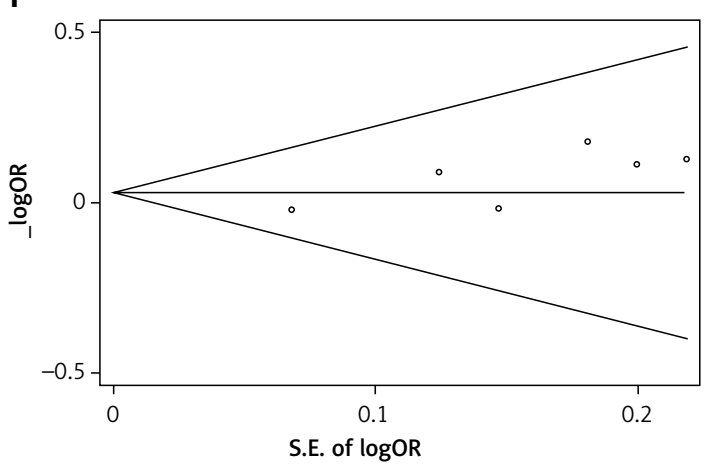

Figure 6. Begg's funnel plot analysis to detect publication bias in eligible studies. Each point represents a separate study for the indicated association. Log $[O R]$, natural logarithm of OR. Horizontal line means effect size. A - AO, B - rs1800450, C - rs1800451, D - rs5030737, E - rs7095891, F - rs7096206, G - rs 11003125

based on different ethnicities be performed to re-evaluate the association. Moreover, additional future studies should include more detailed information concerning the potential confounding factors and multiple SNPs to extend our investigations. 


\section{Acknowledgments}

Yan Cao and Xinjing Wang contributed equally to this work.

Supported by key project of $309^{\text {th }}$ Hospital to Y.C. (2014ZD-004). The funders had no role in study design, data collection and analysis, decision to publish, or preparation of the manuscript.

\section{Conflict of interest}

The authors declare no conflict of interest.

\section{References}

1. World Health Organization. WHO global tuberculosis report. Geneva, Switzerland: WHO, 2013.

2. Hill AV. Aspects of genetic susceptibility to human infectious diseases. Annu Rev Genet 2006; 40: 469-86.

3. Liu Q, Li W, Li D, Feng Y, Tao C. TIRAP C539T polymorphism contributes to tuberculosis susceptibility: evidence from a meta-analysis. Infect Genet Evol 2014; 27: 32-9.

4. Haussler MR, Whitfield GK, Hausler CA. The nuclear vitamin D receptor: biological and molecular regulatory properties revealed. J Bone Miner Res 1998; 13: 324-49.

5. Singla N, Gupta D, Joshi A, Batra N, Singh J. Genetic polymorphisms in the $\mathrm{P} 2 \mathrm{X} 7$ gene and its association with susceptibility to tuberculosis. Int J Tuberc Lung Dis 2012; 16: 224-9.

6. Chen M, Deng J, Su C, et al. Impact of passive smoking, cooking with solid fuel exposure, and MBL/MASP-2 gene polymorphism upon susceptibility to tuberculosis. Int J Infect Dis 2014; 29: 1-6.

7. Matsushita M, Endo Y, Fujita T. Cutting edge: complement-activating complex of ficolin and mannose-binding lectin-associated serine protease. J Immunol 2000; 164: 2281-4.

8. Weitzel T, Zulantay I, Danquah I, et al. Mannose-binding lectin and Toll-like receptor polymorphisms and Chagas disease in Chile. Am J Trop Med Hyg 2012; 86: 229-32.

9. You HL, Lin TM, Wang JC, et al. Mannose-binding lectin gene polymorphisms and mycobacterial lymphadenitis in young patients. Pediatr Infect Dis J 2013; 32: 1005-9.

10. Madsen HO, Garred P, Thiel S, et al. Interplay between promoter and structural gene variants control basal serum level of mannan-binding protein. J Immunol 1995; 155: 3013-20.

11. Thakkinstian A, McElduff P, D'Este C, et al. A method for meta-analysis of molecular association studies. Stat Med 2005; 24: 1291-306.

12. Mantel N, Haenszel W. Statistical aspects of the analysis of data from retrospective studies of disease. J Natl Cancer Inst 1959; 22: 719-48.

13. DerSimonian R, Laird N. Meta-analysis in clinical trials. Control Clin Trials 1986; 7: 177-88.

14. Begg CB, Mazumdar M. Operating characteristics of a rank correlation test for publication bias. Biometrics 1994; 50: 1088-101.

15. Egger M, Davey Smith G, Schneider M, Minder C. Bias in meta-analysis detected by a simple, graphical test. BMJ 1997; 315: 629-34.

16. Chen M, Liang Y, Li W, et al. Impact of MBL and MASP-2 gene polymorphism and its interaction on susceptibility to tuberculosis. BMC Infect Dis 2015; 25: 151.
17. Chen M, Deng J, Su C, et al. Impact of passive smoking, cooking with solid fuel exposure, and MBL/MASP-2 gene polymorphism upon susceptibility to tuberculosis. Int J Infect Dis 2014; 29: 1-6.

18. García-Gasalla M, Milá Llambí J, Losada-López I, et al. Mannose-binding lectin exon 1 and promoter polymorphisms in tuberculosis disease in a Mediterranean area. Int J Immunogenet 2014; 41: 306-11.

19. da Cruz HL, da Silva RC, Segat L, et al. MBL2 gene polymorphisms and susceptibility to tuberculosis in a northeastern Brazilian population. Infect Genet Evol 2013; 19: 323-9.

20. Araújo MS, Graça ES, Azevedo VN, et al. No evidence of association between MBL2A/O polymorphisms and Mycobacterium tuberculosis infection in populations from the Brazilian Amazon region. Hum Immunol 2013; 74: 82-4.

21. Singla N, Gupta D, Joshi A, et al. Association of mannose-binding lectin gene polymorphism with tuberculosis susceptibility and sputum conversion time. Int J Immunogenet 2012; 39: 10-4.

22. Thye T, Niemann S, Walter K, et al. A genetic variant in the promoter region of Toll-like receptor 9 and cervical cancer susceptibility. PLoS One 2011; 6: e20908.

23. de Wit E, van der Merwe L, van Helden PD, Hoal EG. Gene-gene interaction between tuberculosis candidate genes in a South African population. Mamm Genome 2011; 22: 100-10.

24. Li Y, Wu F, Zhang L, Zhang WJ. Association between polymorphism of MBL gene with susceptibility to tuberculosis in Han Population in Xinjiang. Chin J Zoonoses 2011; 27: 769-73.

25. Capparelli R, lannaccone M, Palumbo D, et al. Role played by human mannose-binding lectin polymorphisms in pulmonary tuberculosis. J Infect Dis 2009; 199: 666-72.

26. Cosar H, Ozkinay F, Onay H, et al. Low levels of mannose-binding lectin confers protection against tuberculosis in Turkish children. Eur J Clin Microbiol Infect Dis 2008; 27: 1165-9.

27. Alagarasu K, Selvaraj P, Swaminathan S, et al. Mannose binding lectin gene variants and susceptibility to tuberculosis in HIV-1 infected patients of South India. Tuberculosis (Edinb) 2007; 87: 535-43.

28. Søborg C, Andersen AB, Range N, et al. Influence of candidate susceptibility genes on tuberculosis in a high endemic region. Mol Immunol 2007; 44: 2213-20.

29. Liu W, Zhang F, Xin ZT, et al. Sequence variations in the $M B L$ gene and their relationship to pulmonary tuberculosis in the Chinese Han population. Int J Tuberc Lung Dis 2006; 10: 1098-103.

30. Selvaraj P, Jawahar MS, Rajeswari DN, et al. Role of mannose binding lectin gene variants on its protein levels and macrophage phagocytosis with live Mycobacterium tuberculosis in pulmonary tuberculosis. FEMS Immunol Med Microbiol 2006; 46: 433-7.

31. Garcia-Laorden MI, Pena MJ, Caminero JA, et al. Influence of mannose-binding lectin on HIV infection and tuberculosis in a Western-European population. Mol Immunol 2006; 43: 2143-50.

32. Fitness J, Floyd S, Warndorff DK, et al. Large-scale candidate gene study of tuberculosis susceptibility in the Karonga district of northern Malawi. Am J Trop Med Hyg 2004; 71: 341-9.

33. Søborg C, Madsen HO, Andersen AB, et al. Mannose-binding lectin polymorphisms in clinical tuberculosis. J Infect Dis 2003; 188: 777-82. 
34. Ozbaş-Gerçeker F, Tezcan I, Berkel Al, et al. The effect of mannose-binding protein gene polymorphisms in recurrent respiratory system infections in children and lung tuberculosis. Turk J Pediatr 2003; 45: 95-8.

35. Selvaraj P, Kurian SM, Uma H, Reetha AM, Narayanan PR. Influence of non-MHC genes on lymphocyte response to Mycobacterium tuberculosis antigens; tuberculin reactive status in pulmonary tuberculosis. Indian J Med Res 2000; 112: 86-92.

36. Selvaraj P, Narayanan PR, Reetha AM. Association of functional mutant homozygotes of the mannose binding protein gene with susceptibility to pulmonary tuberculosis in India. Tuber Lung Dis 1999; 79: 221-7.

37. Bellamy R, Ruwende C, Mcadam KP, et al. Mannose binding protein deficiency is not associated with malaria, hepatitis B carriage nor tuberculosis in Africans. Q J Med 1998; 91: 13-8.

38. Denholm JT, McBryde ES, Eisen DP. Mannose-binding lectin and susceptibility to tuberculosis: a meta-analysis. Clin Exp Immunol 2010; 162: 84-90. 